Supporting Information for:

\title{
C-H Amination of Arenes with Hydroxylamine
}

Yi Yang See, Melanie S. Sanford*

Department of Chemistry, University of Michigan, 930 N. University Avenue, Ann Arbor, Michigan 48109, United States

\section{Corresponding Author:}

E-mail: mssanfor@umich.edu 


\section{SUPPORTING INFORMATION}

Table of Contents



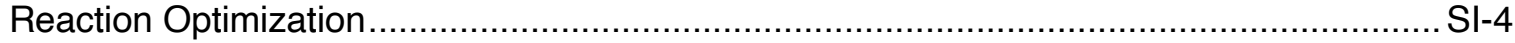

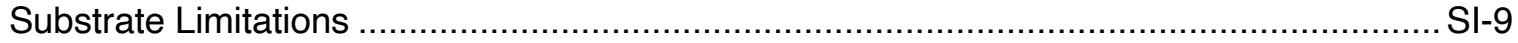

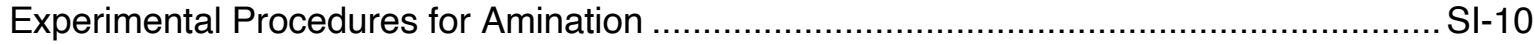

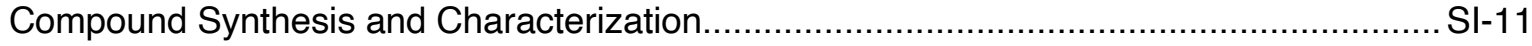

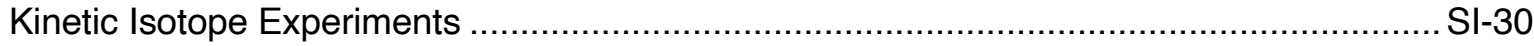

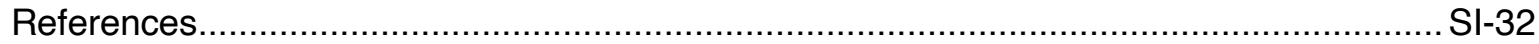

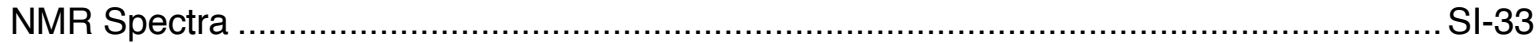




\section{General Information}

NMR spectra were obtained on a Varian MR400 (400.52 MHz for ${ }^{1} \mathrm{H} ; 100.71 \mathrm{MHz}$ for $\left.{ }^{13} \mathrm{C}\right)$, a Varian vnmrs $500\left(500.01 \mathrm{MHz}\right.$ for ${ }^{1} \mathrm{H} ; 125.75 \mathrm{MHz}$ for $\left.{ }^{13} \mathrm{C}\right)$ or a Varian vnmrs $700 \mathrm{MHz}\left(699.76 \mathrm{MHz}\right.$ for ${ }^{1} \mathrm{H}$; $175.95 \mathrm{MHz}$ for ${ }^{13} \mathrm{C}$ ) spectrometer. ${ }^{1} \mathrm{H}$ and ${ }^{13} \mathrm{C}$ chemical shifts are reported in parts per million (ppm) relative to the residual solvent peak $\left(\mathrm{CDCl}_{3}:{ }^{1} \mathrm{H} \mathrm{NMR}=7.26,{ }^{13} \mathrm{C} \mathrm{NMR}=77.16\right.$, Methanol- $d_{4}:{ }^{1} \mathrm{H} \mathrm{NMR}$ $\left.=3.31,{ }^{13} \mathrm{C} \mathrm{NMR}=49.15\right)$. ${ }^{1} \mathrm{H}$ multiplicities are reported as follows: singlet $(\mathrm{s})$, broad singlet (br), doublet (d), triplet (t), quartet (q), pentet (pent) multiplet (m), sextet (sext), septet (sept), doublet of doublets (dd), doublet of triplets (dt), triplet of doublets (td), doublet of doublet of doublets (ddd), doublet of doublet of triplets (ddt). Coupling constants $(\mathcal{J})$ are reported in Hz. GCMS analysis was performed on a Shimadzu GCMSQP2010 gas chromatograph mass spectrometer. The products were separated on a $30 \mathrm{~m}$ length $\times 0.25 \mathrm{~mm}$ id RESTEK XTI-5 column coated with a $0.25 \mu \mathrm{m}$ film. Helium was employed as the carrier gas with a constant column flow of $1.5 \mathrm{~mL} / \mathrm{min}$. The injector temperature was held constant at $250^{\circ} \mathrm{C}$. The GC oven temperature program for low molecular weight compounds was as follows: $32{ }^{\circ} \mathrm{C}$ hold $5 \mathrm{~min}$, ramp $15^{\circ} \mathrm{C} / \mathrm{min}$ to $250{ }^{\circ} \mathrm{C}$, hold for $1.5 \mathrm{~min}$. Melting points were determined with a MelTemp 3.0 (Laboratory Devices, Inc) and are uncorrected. High-resolution mass spectra were recorded on a Micromass AutoSpec Ultima Magnetic Sector mass spectrometer.

All reactions were performed using oven-dried $\left(150^{\circ} \mathrm{C}\right)$ round-bottomed flasks or new reaction vessels unless otherwise stated. Reactions were carried out under an inert atmosphere of nitrogen with dry solvents, unless otherwise stated. Dichloromethane, pentane, diethyl ether, ethyl acetate, toluene, acetonitrile and hexanes were obtained from Fisher Scientific and used as received. $\mathrm{CDCl}_{3}$ was purchased from Cambridge Isotope Laboratories. Anhydrous acetonitrile was purchased from SigmaAldrich and used as received. Titanium trichloride $(12 \%$ in aqueous $\mathrm{HCl})$ was purchased from SigmaAldrich and transferred to a Schlenk flask for long term storage. Titanium(III) sulfate (20\% in $1-4 \%$ sulfuric acid) was purchased from Alfa Aesar. Sulfuric acid 95-98\% was purchased from VWR Chemicals (supplier BDH) and used as received.

Yields refer to chromatographically and spectroscopically ( ${ }^{1} \mathrm{H}$ NMR) homogeneous materials, unless otherwise stated. Reagents were purchased at the highest commercial quality and used without further purification, unless otherwise stated. Reactions were monitored by thin-layer chromatography carried out on $0.25 \mathrm{~mm}$ E. Merck silica gel plates (60F-254) using ultraviolet light as a visualizing agent and an acidic mixture of $p$-anisaldehyde or a basic mixture of potassium permanganate and heat as developing agents. 


\section{Reaction Optimization}

\section{Mediator Screen}

Hydroxylamine hydrochloride $(0.3 \mathrm{mmol})$ and the corresponding metal salt were added to a reaction vial and placed under an atmosphere of nitrogen. A solution of anisole $(0.10 \mathrm{mmol})$ in acetonitrile $(0.50$ $\mathrm{mL}$ ) was added in 1 portion, and the reaction was stirred vigorously under nitrogen for $24 \mathrm{~h}$ at rt (25 $\left.{ }^{\circ} \mathrm{C}\right)$. After this time, the reaction was basified with sodium hydroxide $(20 \%)$ and extracted with ethyl acetate. The internal standard 1,3,5-trimethoxybenzene was added, and the crude mixture was analyzed by GC.

\begin{tabular}{|c|c|c|c|}
\hline 6 & $\begin{array}{c}+\quad \mathrm{NH}_{2} \mathrm{OH} \cdot \mathrm{HCl} \\
\\
(3.0 \text { equiv })\end{array}$ & $\begin{array}{c}\text { metal salt ( } 0.5 \text { equiv) } \\
\operatorname{MeCN}(c=0.1 \mathrm{M}), 25^{\circ} \mathrm{C} \\
24 \mathrm{~h}\end{array}$ & $\nabla$ \\
\hline Metal & & Salts & Yield $(\%)^{b}$ \\
\hline $\mathrm{Fe}(\mathrm{II})$ & $\mathrm{FeF}_{2}, \mathrm{FeCl}_{2}, \mathrm{FeBr}_{2}$ & e(oxalate $)_{2}, \mathrm{Fe}(\mathrm{OAc})_{2}, \mathrm{FeSO}_{4}, \mathrm{Fe}\left(\mathrm{BF}_{4}\right)_{2}$, ferrocene & 0 \\
\hline $\mathrm{Fe}(\mathrm{III})$ & $\mathrm{Fe}_{2}\left(\mathrm{SO}_{4}\right)_{3}, \mathrm{FeF}_{3}, \mathrm{~F}$ & $r_{3}, \mathrm{Fe}(\mathrm{OTf})_{3}, \mathrm{Fe}(\mathrm{acac})_{3}, \mathrm{Fe}\left(\mathrm{NO}_{3}\right)_{3}$ & 0 \\
\hline $\mathrm{Cu}(\mathrm{l})$ & $\mathrm{Cu}[\mathrm{MeCN}]_{4} \mathrm{OTf}, \mathrm{Cu}$ & $\mathrm{leCN}]_{4} \mathrm{PF}_{6}, \mathrm{CuCl}, \mathrm{Cul}$ & 0 \\
\hline $\mathrm{Cu}(\mathrm{II})$ & $\mathrm{CuF}_{2}, \mathrm{CuCl}_{2}, \mathrm{CuBr}_{\mathrm{s}}$ & $\mathrm{Cu}(\mathrm{OAc})_{2}, \mathrm{CuO}$ & 0 \\
\hline $\mathrm{Mn}(\mathrm{II})$ & $\mathrm{MnCl}_{2}, \mathrm{Mn}(\mathrm{OAc})_{2}$ & $(\mathrm{OTf})_{2}, \mathrm{MnSO}_{4}$ & 0 \\
\hline $\mathrm{Mn}(\mathrm{III})$ & $\mathrm{Mn}(\mathrm{OAc})_{3}$ & & 0 \\
\hline $\mathrm{Cr}$ & $\mathrm{CrCl}_{2}, \mathrm{CrCl}_{3}$ & & 0 \\
\hline Co & $\mathrm{CoCl}_{2}, \mathrm{CoBr}_{2}, \mathrm{Co}(\mathrm{E}$ & $\left.{ }_{4}\right)_{2}, \mathrm{CoF}_{3}$ & 0 \\
\hline $\mathrm{Ni}(\mathrm{II})$ & $\mathrm{NiCl}_{2}, \mathrm{Ni}(\mathrm{OAc})_{2}, \mathrm{Ni}$ & $\mathrm{cac})_{2}, \mathrm{Ni}(\mathrm{OTf})_{2}, \mathrm{Ni}\left(\mathrm{BF}_{4}\right)_{2}$ & 0 \\
\hline \multirow[t]{2}{*}{$\mathrm{Ti}(\mathrm{III})$} & $\mathrm{Cp}_{2} \mathrm{TiCl}^{\mathrm{C}}$ & & 0 \\
\hline & $\mathrm{TiCl}_{3}(\mathrm{THF})_{3}{ }^{\mathrm{c}}$ & & $13 \%$ \\
\hline
\end{tabular}

a Conditions: substrate $(0.1 \mathrm{mmol}), \mathrm{NH}_{2} \mathrm{OH} \cdot \mathrm{HCl}(0.3 \mathrm{mmol}), \mathrm{MeCN}(0.5 \mathrm{~mL})$, metal salt $(0.05 \mathrm{mmol})$, room temperature, $24 \mathrm{~h} ;{ }^{b}$ determined by GC using 1,3,5-trimethoxybenzene as internal standard; ${ }^{c} 1$ equiv of Ti(III) used * significant amount of bromo-anisole observed by GCMS 


\section{Additional Optimization}

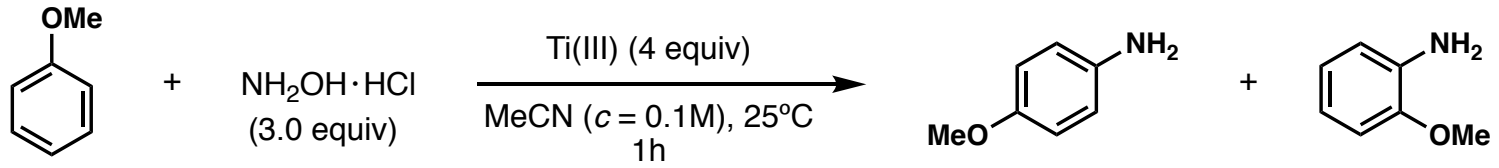

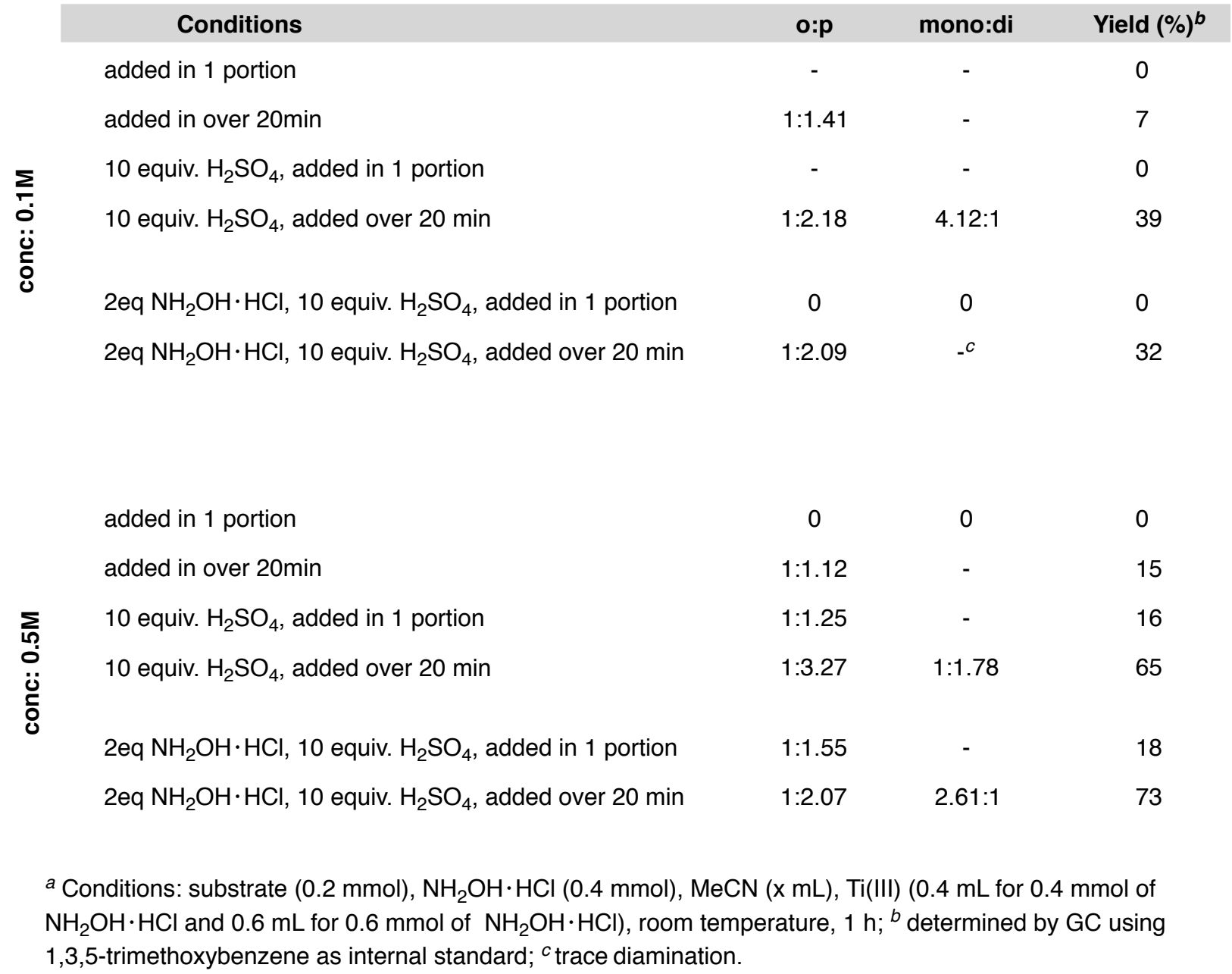

Note: yields refer to the combined yield of mono and di-aminated products

Summary: The reaction yield improved with increasing reaction concentration and slow introduction of the titanium solution. The addition of sulfuric acid was found to be critical to ensure good yields of the product. Visually, the presence of sulfuric acid significantly reduced the decolorization of the titanium solution and the amount of titanium solution needed to reach the purple end-point color. Excess hydroxylamine in this case resulted in significant over reaction, which reduced the yield of the desired product. 


\section{Acid Equivalent Screen:}

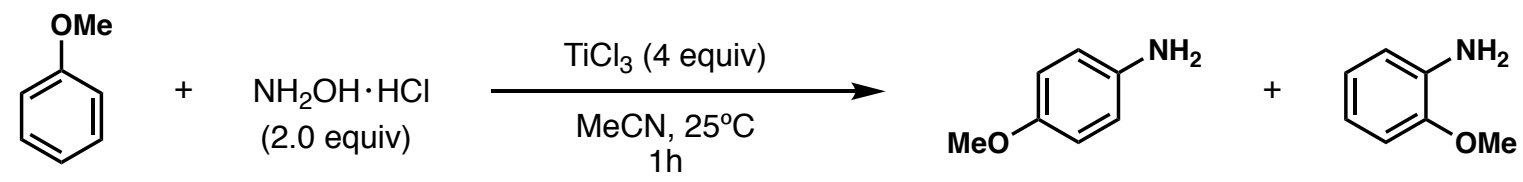

$\begin{array}{lcccc}\text { Acid } & \text { [anisole] } & \text { o:p ratio } & \text { mono:diamination } & \text { Yield (\%) } \\ \mathrm{H}_{2} \mathrm{SO}_{4}, 1 \text { equiv } & 0.50 \mathrm{M} & 1: 1.92 & - & 15 \\ \mathrm{H}_{2} \mathrm{SO}_{4}, 2 \text { equiv } & 0.50 \mathrm{M} & 1: 2.17 & 4.5: 1 & 25 \\ \mathrm{H}_{2} \mathrm{SO}_{4}, 5 \text { equiv } & 0.50 \mathrm{M} & 1: 2.21 & 1.9: 1 & 34 \\ \mathrm{H}_{2} \mathrm{SO}_{4}, 10 \text { equiv } & 0.50 \mathrm{M} & 1: 2.07 & \mathbf{2 . 6 : 1} & 73 \\ \mathrm{H}_{2} \mathrm{SO}_{4}, 15 \text { equiv } & 0.50 \mathrm{M} & 1: 1.96 & 2.6: 1 & 66 \\ \mathrm{H}_{2} \mathrm{SO}_{4}, 20 \text { equiv } & 0.50 \mathrm{M} & 1: 2.16 & 1.6: 1 & 40\end{array}$

a Conditions: substrate $(0.2 \mathrm{mmol}), \mathrm{NH}_{2} \mathrm{OH} \cdot \mathrm{HCl}(0.4 \mathrm{mmol}), \mathrm{MeCN}(\mathrm{x} \mathrm{mL}), \mathrm{TiCl}_{3}(0.4 \mathrm{~mL}$, added over 20min), room temperature, $1 \mathrm{~h} ;{ }^{b}$ determined by GC using 1,3,5-trimethoxybenzene as internal standard

\section{$\underline{\text { Reaction Concentration Screen: }}$}

\begin{tabular}{|c|c|c|c|c|}
\hline Acid & [anisole] & o:p ratio & mono:diamination & Yield $(\%)^{b}$ \\
\hline $\mathrm{H}_{2} \mathrm{SO}_{4}, 10$ equiv & $0.10 \mathrm{M}$ & $1: 2.09$ & - & 34 \\
\hline $\mathrm{H}_{2} \mathrm{SO}_{4}, 10$ equiv & $0.25 \mathrm{M}$ & $1: 2.04$ & $2.8: 1$ & 51 \\
\hline $\mathrm{H}_{2} \mathrm{SO}_{4}, 10$ equiv & $0.50 \mathrm{M}$ & 1:2.07 & 2.6:1 & 73 \\
\hline $\mathrm{H}_{2} \mathrm{SO}_{4}, 10$ equiv & $0.75 \mathrm{M}$ & $1: 2.08$ & $2.1: 1$ & 65 \\
\hline $\mathrm{H}_{2} \mathrm{SO}_{4}, 10$ equiv & $1.0 \mathrm{M}$ & $1: 2.17$ & $2.7: 1$ & 51 \\
\hline
\end{tabular}

Note: yields refer to the combined yield of mono and di-aminated products 


\section{Solvent and Acid Screens}

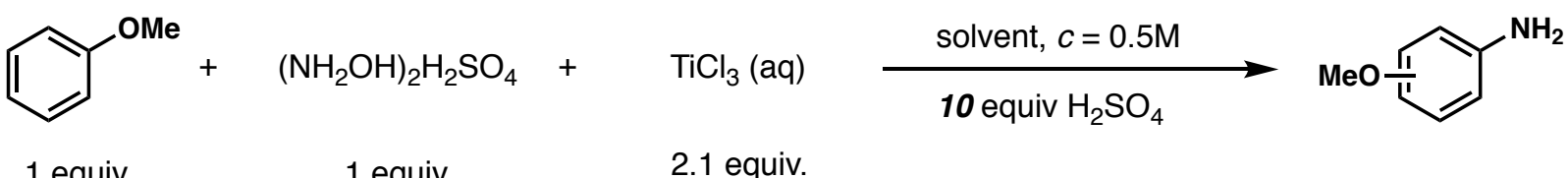

1 equiv.

1 equiv.

$\begin{array}{cccccc}\text { Solvent } & \text { Mono (\%) } & \text { o:p ratio } & \text { diamination (\%) } & \text { Total }^{\boldsymbol{b}}(\%) & \text { Comments } \\ \mathrm{AcOH} / \mathrm{H}_{2} \mathrm{O}(5: 2) & 54 & 1: 2.2 & 21 & 76 & \\ \mathrm{MeCN} & 48 & 1: 2.1 & 24 & 73 & \\ \mathrm{HFIP} & - & - & - & - & \text { no SM or pdt by GC } \\ \mathrm{MeOH}^{*} & 30 & 1: 3.3 & - & 30 & \\ \mathrm{DMF}^{*} & 36 & 1: 5.1 & - & 36 & \\ \mathrm{THF}^{*} & 18 & 1: 1.4 & - & 4 & \end{array}$

Conditions: substrate $(0.2 \mathrm{mmol}), \mathrm{NH}_{2} \mathrm{OH} \cdot \mathrm{HCl}(0.4 \mathrm{mmol})$, solvent $(0.4 \mathrm{~mL})$, conc. $\mathrm{H}_{2} \mathrm{SO}_{4}(2.0 \mathrm{mmol}), \mathrm{TiCl}_{3}$ ( $0.4 \mathrm{~mL}$, added over $20 \mathrm{~min}$ ), room temperature, $1 \mathrm{~h}$; ${ }^{a}$ solvents used was not dried or degassed previously; ${ }^{b}$ determined by GC using 1,3,5-trimethoxybenzene as internal standard; * these solvents are known to react with aminyl radicals to form carbon-based radicals

Note: yields refer to the combined yield of mono and di-aminated products

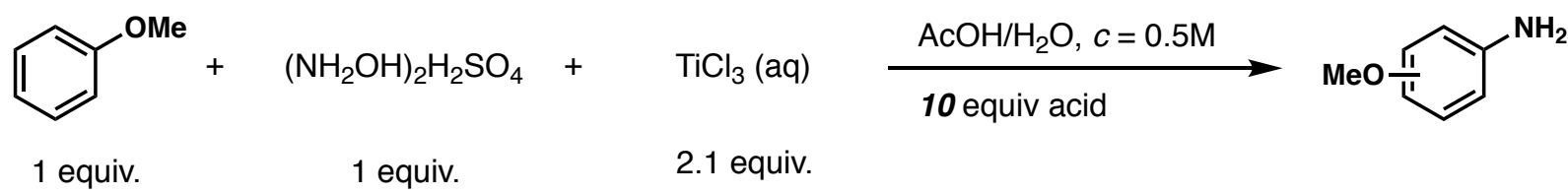

$\begin{array}{ccc}\text { Acid } & \text { mono (\%) } & \text { o:p rati } \\ \mathrm{H}_{2} \mathrm{SO}_{4} & 54 & 1: 2.2 \\ \mathrm{H}_{3} \mathrm{PO}_{4} & 9 & 1: 4.1 \\ \mathrm{HCl} & 15 & 1: 1.3 \\ \mathrm{HBr} & - & - \\ \mathrm{HBF}_{4} & 5 & 1: 2.8 \\ \mathrm{TfOH} & 5 & 1: 0.6 \\ \mathrm{TFA} & 3 & \text { ortho } \\ \mathrm{TsOH} & 3 & 1: 1.9 \\ \mathrm{MsOH} & 31 & 1: 2.1\end{array}$

Conditions: substrate $(0.2 \mathrm{mmol}), \mathrm{NH}_{2} \mathrm{OH} \cdot \mathrm{HCl}(0.4 \mathrm{mmol})$, solvent $(0.4 \mathrm{~mL})$, acid $(2.0 \mathrm{mmol}), \mathrm{TiCl}_{3}(0.4 \mathrm{~mL}$, added over 20min), room temperature, $1 \mathrm{~h} ;{ }^{a}$ determined by GC using 1,3,5-trimethoxybenzene as internal standard.

Note: Sulfuric acid was found to be uniquely suitable for this reaction, with other acids (over a range of $p K_{a}$ ) being significantly less effective. 


\section{Screen of Various Ti(III) Solutions and Hydroxylamine Salts}



\begin{tabular}{|c|c|c|c|c|c|c|}
\hline & Ti(III) source & $\left(\mathrm{NH}_{2} \mathrm{OH}\right) \mathrm{HX}$ & mono (\%) & o:p ratio & diamination & Total $^{a}(\%$ \\
\hline \multirow{3}{*}{  } & $\mathrm{Ti}_{2}\left(\mathrm{SO}_{4}\right)_{3}$ & $\left(\mathrm{NH}_{2} \mathrm{OH}\right)_{2} \mathrm{H}_{2} \mathrm{SO}_{4}$ & 3 & $1: 0.5$ & - & 3 \\
\hline & $\mathrm{TiCl}_{3}$ & $\left(\mathrm{NH}_{2} \mathrm{OH}\right)_{2} \mathrm{H}_{2} \mathrm{SO}_{4}$ & 6 & $1: 0.42$ & - & 6 \\
\hline & $\mathrm{TiCl}_{3}$ & $\left(\mathrm{NH}_{2} \mathrm{OH}\right) \mathrm{HCl}$ & 4 & $1: 0.2$ & - & 4 \\
\hline \multirow{3}{*}{$\begin{array}{l}O^{+} \\
\mathscr{N}_{N} \\
\mathbf{I} \\
\dot{3} \\
\frac{3}{0} \\
0\end{array}$} & $\mathrm{Ti}_{2}\left(\mathrm{SO}_{4}\right)_{3}$ & $\left(\mathrm{NH}_{2} \mathrm{OH}\right)_{2} \mathrm{H}_{2} \mathrm{SO}_{4}$ & 25 & $1: 3.7$ & 2 & 28 \\
\hline & $\mathrm{TiCl}_{3}$ & $\left(\mathrm{NH}_{2} \mathrm{OH}\right)_{2} \mathrm{H}_{2} \mathrm{SO}_{4}$ & 48 & $1: 2.6$ & 16 & 65 \\
\hline & $\mathrm{TiCl}_{3}$ & $\left(\mathrm{NH}_{2} \mathrm{OH}\right) \mathrm{HCl}$ & 54 & $1: 2.2$ & 21 & 76 \\
\hline
\end{tabular}

Conditions: substrate $(0.2 \mathrm{mmol}), \mathrm{NH}_{2} \mathrm{OH} \cdot \mathrm{HCl}(0.4 \mathrm{mmol})$, solvent $(\mathrm{x} \mathrm{mL}), \mathrm{TiCl}_{3}(0.4 \mathrm{~mL}$, added over $20 \mathrm{~min})$, room temperature, $1 \mathrm{~h}$; ${ }^{a}$ determined by GC using 1,3,5-trimethoxybenzene as internal standard.

Notes: Commercial titanium(III) chloride (in hydrochloric acid) was found to be optimal, although titanium(III) sulfate was also effective. Interestingly, hydroxylamine sulfate gave consistently lower yields compared to hydroxylamine hydrochloride.

Yields refer to the combined yield of mono and di-aminated products

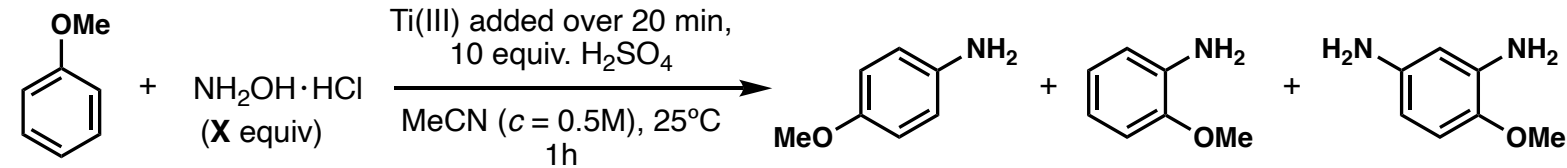

\begin{tabular}{|c|c|c|c|c|c|c|}
\hline $\mathrm{NH}_{2} \mathrm{OH} \cdot \mathrm{HCl}$ Equivalence & ortho $(\%)^{b}$ & para $(\%)^{b}$ & di-amine $(\%)^{b}$ & $0: p$ & mono:di & Total \\
\hline 2eq $\mathrm{NH}_{2} \mathrm{OH} \cdot \mathrm{HCl}$ & 18 & 36 & 20 & $1: 2.07$ & $2.61: 1$ & 73 \\
\hline 3eq $\mathrm{NH}_{2} \mathrm{OH} \cdot \mathrm{HCl}$ & 8 & 22 & 38 & $1: 2.70$ & $1: 1.27$ & 67 \\
\hline 4eq $\mathrm{NH}_{2} \mathrm{OH} \cdot \mathrm{HCl}$ & 4 & 14 & 57 & $1: 3.40$ & $1: 3.08$ & 75 \\
\hline
\end{tabular}

${ }^{a}$ Conditions: substrate $(0.2 \mathrm{mmol}), \mathrm{NH}_{2} \mathrm{OH} \cdot \mathrm{HCl}(\mathrm{x} \mathrm{mmol}), \mathrm{MeCN}(0.4 \mathrm{~mL}), \mathrm{Ti}(\mathrm{III})(0.4 \mathrm{~mL}$ for $0.4 \mathrm{mmol}$ of $\mathrm{NH}_{2} \mathrm{OH} \cdot \mathrm{HCl}, 0.6 \mathrm{~mL}$ for $0.6 \mathrm{mmol}$ of $\mathrm{NH}_{2} \mathrm{OH} \cdot \mathrm{HCl}, 0.7 \mathrm{~mL}$ for $0.8 \mathrm{mmol}$ of $\mathrm{NH}_{2} \mathrm{OH} \cdot \mathrm{HCl}$ ), room temperature, 1 $\mathrm{h} ;{ }^{b}$ determined by GC using 1,3,5-trimethoxybenzene as internal standard. 


\section{Substrate Limitations}

The following substrates were examined under the standard amination conditions. However, low conversion were noted.

Low conversions (<30\%):<smiles>c1ccccc1</smiles>

10\% conversion<smiles>Nc1ccc(Oc2ccc(Br)cc2)cc1</smiles>

$\sim 10 \%$ conversion<smiles>COc1ccc(N)c(OC)c1Br</smiles>

$\sim 21 \%$ conversion

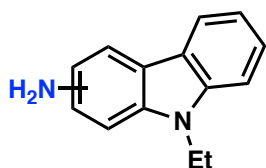

$\sim 10 \%$ conversion mono + diamine

No reactions/incompatibility:<smiles>Cc1cc(=O)cc(C)o1</smiles>

$<5 \%$ conversion<smiles>Brc1ccccc1</smiles>

0\% conversion

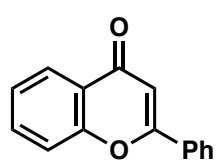

$0 \%$ conversion<smiles>c1ccc2scnc2c1</smiles>

$0 \%$ conversion

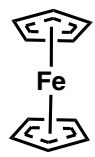

$0 \%$ conversion<smiles>c1ccc2[nH]cnc2c1</smiles>

$0 \%$ conversion<smiles>Cn1ccccc1=O</smiles>

$0 \%$ conversion<smiles>[AsH3-]n1cccn1</smiles>

$100 \%$ conversion; no desired amination<smiles>c1ccc2[nH]ncc2c1</smiles>

$100 \%$ conversion; no desired amination<smiles>CC(C)Cn1cc(Br)cn1</smiles>

$0 \%$ conversion; no desired amination 


\section{Experimental Procedures for Amination}

General Procedure for Amination



The appropriate arene $(1.0 \mathrm{mmol})$ and hydroxylamine hydrochloride $(2.0$ or $3.0 \mathrm{mmol})$ were suspended in acetonitrile $(2.0 \mathrm{~mL})$. The reaction was immersed in a rt $\left(25^{\circ} \mathrm{C}\right)$ water bath. Concentrated sulfuric acid $(10 \mathrm{mmol})$ was added dropwise, and the reaction was stirred for $10 \mathrm{~min}$ open to air. After this time, the reaction was sealed with a rubber septum, and the headspace was purged for 1 min with nitrogen. Titanium trichloride $(12 \%$ in aqueous $\mathrm{HCl}$ ) was added via a syringe pump (addition rate of 4 $\mathrm{mL} / \mathrm{h}$ ) until the purple color of the titanium trichloride solution persisted for more than $10 \mathrm{~min}$. The reaction was stirred for an additional $10 \mathrm{~min}$ before the addition of saturated aqueous sodium citrate ( $3 \mathrm{~mL}$ ). Sodium hydroxide ( $20 \%$ aqueous) was then added slowly until the reaction mixture became basic, and the resulting solution was then extracted with ethyl acetate $(5 \times 10 \mathrm{~mL})$. The combined organic phases were dried over magnesium sulfate and purified by column chromatography over silica. 


\title{
Compound Synthesis and Characterization
}

\author{
$N$-phenylacetamide (SI-1) \\ ${ }^{N}{ }^{\mathrm{N}}$
}

A modification of the general procedure was followed using benzene (156 mg, $2.0 \mathrm{mmol}, 2.0$ equiv) and hydroxylamine hydrochloride $(69.5 \mathrm{mg}, 1.0 \mathrm{mmol}, 1.0$ equiv) as starting materials. After concentrating via rotary evaporation, the reaction was dissolved in dichloromethane $(5.0 \mathrm{~mL})$ and treated with acetic anhydride (1.5 equiv) and triethylamine (2.0 equiv) at rt for $5 \mathrm{~h}$. Then, the reaction was quenched with saturated sodium bicarbonate $(3 \mathrm{~mL})$ and extracted with dichloromethane $(3 \times 5$ $\mathrm{mL})$, and the orgaic extracts were dried over magnesium sulfate. Purification by column chromatography (hexanes/EtOAc 4:1) afforded the product as a cream colored solid (13 mg, 10\%).

1H NMR $\left(500 \mathrm{MHz}, \mathrm{CDCl}_{3}\right): \delta 7.50(\mathrm{~d}, J=8.0 \mathrm{~Hz}, 2 \mathrm{H}), 7.44(\mathrm{br} s, 1 \mathrm{H}), 7.31(\mathrm{t}, J=7.7 \mathrm{~Hz}, 2 \mathrm{H}), 7.10$ $(\mathrm{t}, J=7.4 \mathrm{~Hz}, 1 \mathrm{H}), 2.16(\mathrm{~s}, 3 \mathrm{H})$.

${ }^{13} \mathrm{CNMR}\left(126 \mathrm{MHz}, \mathrm{CDCl}_{3}\right): \delta 168.5,138.0,129.1,124.4,120.0,24.7$.

HRMS: (ESI) m/z [M+H]+ calcd. for $\mathrm{C}_{8} \mathrm{H}_{10} \mathrm{NO}+136.0757$; found 136.0755 .

The characterization data matched that of the commercial sample.

\section{2-methoxyaniline (2b), 4-methoxyaniline (2a), 4-methoxybenzene-1,3-diamine (2c)<smiles>COc1ccc(N)cc1</smiles>

The general procedure was followed using anisole (108 $\mathrm{mg}, 1.0 \mathrm{mmol}$ ) and hydroxylamine hydrochloride $(139 \mathrm{mg}, 2.0 \mathrm{mmol})$ as starting materials. The crude mixture was purified by flash column chromatography (hexanes/EtOAc 4:1 to 1:1 to 0:1) to afford 2-methoxyaniline as a pale yellow oil (20.1 mg, 16\%), 4-methoxylaniline as a light gray solid (42 mg, 34\%), and 4-methoxybenzene-1,3diamine as a gray solid that darkens rapidly in air (32 $\mathrm{mg}, 23 \%)$.

2-methoxyaniline (2b)<smiles>COc1ccccc1N</smiles>

$\underline{1 \mathrm{H} N M R}\left(500 \mathrm{MHz}, \mathrm{CDCl}_{3}\right):$ $6.84-6.77(\mathrm{~m}, 2 \mathrm{H}), 6.77-6.71(\mathrm{~m}, 2 \mathrm{H}), 3.86(\mathrm{br} \mathrm{s}, 3 \mathrm{H}), 3.79(\mathrm{~s}, 2 \mathrm{H})$.

13C NMR $\left(126 \mathrm{MHz}, \mathrm{CDCl}_{3}\right): \delta 147.4,136.3,121.2,118.6,115.1,110.6,55.5$.

HRMS: (ESI) m/z [M+H]+ calcd. for $\mathrm{C}_{7} \mathrm{H}_{10} \mathrm{NO}+124.0757$; found 124.0756 .

4-methoxyaniline (2a) 
OMe

1H NMR $\left(500 \mathrm{MHz}, \mathrm{CDCl}_{3}\right): \delta$ 6.78-6.71 (m, 2H), 6.69-6.61 (m, 2H), $3.75(\mathrm{~s}, 3 \mathrm{H}), 3.42(\mathrm{br} \mathrm{s}, 2 \mathrm{H})$.

$\underline{13 \mathrm{C} \mathrm{NMR}}\left(126 \mathrm{MHz}, \mathrm{CDCl}_{3}\right): \delta 152.9,140.1,116.5,114.9,55.9$.

HRMS: (ESI) m/z [M+H]+ calcd. for $\mathrm{C}_{7} \mathrm{H}_{10} \mathrm{NO}+124.0757$; found 124.0759.

The characterization data matched that found in the literature (ACS Catal. 2016, 6, 8162-8165).

4-methoxybenzene-1,3-diamine (2c)<smiles>COc1ccc(N)cc1N</smiles>

1H NMR $\left(500 \mathrm{MHz}, \mathrm{CDCl}_{3}\right) \delta 6.61(\mathrm{~d}, J=8.3 \mathrm{~Hz}, 1 \mathrm{H}), 6.13(\mathrm{~d}, J=2.7 \mathrm{~Hz}, 1 \mathrm{H}), 6.07$ (dd, $J=8.4,2.7$ $\mathrm{Hz}, 1 \mathrm{H}), 3.77$ (s, 3H), 3.53 (br s, 4H).

13C NMR $\left(126 \mathrm{MHz}, \mathrm{CDCl}_{3}\right): \delta 141.0,140.8,137.2,112.4,104.9,103.5,56.4$.

HRMS: $(E S I) m / z[M+H]+$ calcd. for $\mathrm{C}_{7} \mathrm{H}_{11} \mathrm{~N}_{2} \mathrm{O}+139.0866$; found 139.0865 .

4-isopropoxyaniline (3a), 4-isopropoxybenzene-1,3-diamine (3b)<smiles>CCOc1ccc(N)cc1</smiles>

The general procedure was followed using isopropoxybenzene $(136 \mathrm{mg}, 1.0 \mathrm{mmol})$ and hydroxylamine hydrochloride $(139 \mathrm{mg}, 2.0 \mathrm{mmol}$ ) as starting materials. The crude mixture was purified by flash column chromatography (hexanes/EtOAc $4: 1$ to $3: 1$ ) to afford 4-isopropoxyaniline $(60.5 \mathrm{mg}, 40 \%)$ first, followed by 4 -isopropoxybenzene-1,3-diamine $(38.7 \mathrm{mg}, 22 \%)$ as light yellow oils that darkened upon storage on the benchtop.

4-isopropoxyaniline (3a)<smiles>CCOc1ccc(N)cc1</smiles>

1H NMR: $\left(500 \mathrm{MHz}, \mathrm{CDCl}_{3}\right): \delta 6.74(\mathrm{~d}, J=8.7 \mathrm{~Hz}, 2 \mathrm{H}), 6.63(\mathrm{~d}, J=8.7 \mathrm{~Hz}, 2 \mathrm{H}), 4.37$ (hept, $J=6.1$ $\mathrm{Hz}, 1 \mathrm{H}), 3.42(\mathrm{br} \mathrm{s}, 2 \mathrm{H}), 1.29(\mathrm{~d}, J=6.1 \mathrm{~Hz}, 6 \mathrm{H})$.

13C NMR: $\left(126 \mathrm{MHz}, \mathrm{CDCl}_{3}\right): \delta 151.0,140.3,118.0,116.5,71.2,22.3$.

HRMS: (ESI) m/z [M+H]+ calcd. for $\mathrm{C}_{9} \mathrm{H}_{14} \mathrm{NO}+152.1070$; found 152.1071.

The characterization data matched that found in the literature (Organometallics 2007, 26, 5627-5635). 
4-isopropoxybenzene-1,3-diamine (3b)<smiles>CCOc1ccc(N)cc1N</smiles>

$\underline{\text { 1H NMR }}\left(500 \mathrm{MHz}, \mathrm{CDCl}_{3}\right): \delta 6.64(\mathrm{~d}, J=8.4 \mathrm{~Hz}, 1 \mathrm{H}), 6.12(\mathrm{~d}, J=2.7 \mathrm{~Hz}, 1 \mathrm{H}), 6.04(\mathrm{dd}, J=8.4,2.7$ $\mathrm{Hz}, 1 \mathrm{H}$ ), 4.31 (hept, $J=6.1 \mathrm{~Hz}, 1 \mathrm{H}), 3.70(\mathrm{br} \mathrm{s}, 1 \mathrm{H}), 3.38(\mathrm{br} \mathrm{s}, 1 \mathrm{H}), 1.30(\mathrm{~d}, J=6.1 \mathrm{~Hz}, 6 \mathrm{H})$.

13C NMR $\left(126 \mathrm{MHz}, \mathrm{CDCl}_{3}\right): \delta 141.3,138.9,138.6,117.2,105.2,103.4,72.3,22.5$.

HRMS: (ESI) m/z [M+H]+ calcd. for $\mathrm{C}_{9} \mathrm{H}_{15} \mathrm{~N}_{2} \mathrm{O}+167.1179$; found 167.1177.

\section{4-(tert-butyl)aniline (4a), 3-(tert-butyl)aniline (4b)}<smiles>CC(C)(C)c1ccc(N)cc1</smiles>

The general procedure was followed using tert-butylbenzene (134 mg, $1.0 \mathrm{mmol})$ and hydroxylamine hydrochloride (139 mg, $2.0 \mathrm{mmol}$ ) as starting materials. The crude oil was purified by flash column chromatography (hexanes/EtOAc $4: 1$ to $3: 1$ ) to afford an isomeric mixture of the title compounds (4a : 4b $1.82: 1)$ as a yellow oil $(77.5 \mathrm{mg}, 52 \%)$.

4-(tert-butyl)aniline (4a)<smiles>Nc1ccc(Br)cc1</smiles>

1H NMR (500 MHz, CDCl $): \delta 7.20(\mathrm{~d}, J=8.5 \mathrm{~Hz}, 2 \mathrm{H}), 6.66(\mathrm{~d}, J=8.5 \mathrm{~Hz}, 2 \mathrm{H}), 3.58(\mathrm{br} \mathrm{s}, 2 \mathrm{H}), 1.29$ $(\mathrm{s}, 9 \mathrm{H})$.

13C NMR $\left(126 \mathrm{MHz}, \mathrm{CDCl}_{3}\right): \delta 143.9,141.5,126.2,115.1,34.0,31.7$.

3-(tert-butyl)aniline (4b)<smiles>CC(C)(C)c1cccc(N)c1</smiles>

1H NMR $\left(500 \mathrm{MHz}, \mathrm{CDCl}_{3}\right): \delta 7.11(\mathrm{t}, J=7.8 \mathrm{~Hz}, 1 \mathrm{H}), 6.82(\mathrm{ddd}, J=7.8,1.9,1.0 \mathrm{~Hz}, 1 \mathrm{H}), 6.74(\mathrm{t}, J=$ $2.1 \mathrm{~Hz}, 1 \mathrm{H}$ ), 6.53 (ddd, $J=7.8,2.3,1.0 \mathrm{~Hz}, 1 \mathrm{H}$ ), 3.58 (br s, 2H), 1.30 (s, 9H).

13C NMR $\left(126 \mathrm{MHz}, \mathrm{CDCl}_{3}\right): \delta 152.6,146.2,129.1,116.0,112.6,112.5,34.7,31.4$.

HRMS: (ESI) m/z [M+H]+ calcd. for $\mathrm{C}_{10} \mathrm{H}_{16} \mathrm{~N}+150.1277$; found 150.1278 .

The characterization data matched that found in the literature (Angew. Chem. Int. Ed. 2019, 58, 526531). 


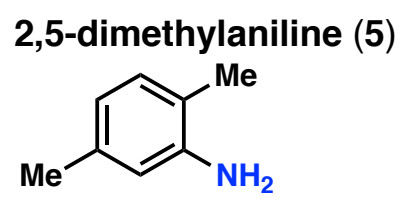

The general procedure was followed using para-xylene (106 mg, $1.0 \mathrm{mmol}$ ) and hydroxylamine hydrochloride $(139 \mathrm{mg}, 2.0 \mathrm{mmol})$ as starting materials. The crude product was purified by flash column chromatography (hexanes/EtOAc 5:1) to afford the title compound (84 $\mathrm{mg}, 70 \%$ ) as a light yellow oil.

1H NMR $\left(500 \mathrm{MHz}, \mathrm{CDCl}_{3}\right): \delta 6.95(\mathrm{~d}, J=7.5 \mathrm{~Hz}, 1 \mathrm{H}), 6.54(\mathrm{~d}, J=7.7 \mathrm{~Hz}, 1 \mathrm{H}), 6.52(\mathrm{~s}, 1 \mathrm{H}), 3.55(\mathrm{br}$ $\mathrm{s}, 2 \mathrm{H}), 2.26(\mathrm{~s}, 3 \mathrm{H}), 2.14(\mathrm{~s}, 3 \mathrm{H})$.

${ }^{13} \mathrm{C} \mathrm{NMR}\left(126 \mathrm{MHz}, \mathrm{CDCl}_{3}\right): \delta 144.5,136.7,130.4,119.5,119.4,115.8,21.2,17.0$.

HRMS: (ESI) m/z [M+H]+calcd. for $\mathrm{C}_{8} \mathrm{H}_{12} \mathrm{~N}+122.0964$; found 122.0963 .

The characterization data matched that found in the literature (ACS Catal. 2016, 6, 8162-8165).

\section{2,4-dimethylaniline (6a), 2,6-dimethylaniline (6b), 3,5-dimethylaniline (6c)}<smiles>Cc1ccc(N)c(C)c1</smiles>

The general procedure was followed using meta-xylene $(106 \mathrm{mg}, 1.0 \mathrm{mmol})$ and hydroxylamine hydrochloride (139 mg, $2.0 \mathrm{mmol})$ as starting materials. The crude product was purified by flash column chromatography (hexanes/EtOAc $5: 1)$ to afford an inseparable mixture (6a : 6b : 6c $7.74: 1.82$ $: 1$ ) of the title compounds $(72 \mathrm{mg}, 60 \%)$ as a light yellow oil. The purified spectra was compared to commercially obtained samples of the individual isomers to confirm their identities.

2,4-dimethylaniline (6a)<smiles>Cc1ccc(N)c(C)c1</smiles>

1H NMR $\left(700 \mathrm{MHz}, \mathrm{CDCl}_{3}\right): \delta 6.90(\mathrm{~d}, J=2.1 \mathrm{~Hz}, 1 \mathrm{H}), 6.87(\mathrm{dd}, J=7.9,2.0 \mathrm{~Hz}, 1 \mathrm{H}), 6.61(\mathrm{~d}, J=7.9$ $\mathrm{Hz}, 1 \mathrm{H}), 3.50(\mathrm{br} \mathrm{s}, 2 \mathrm{H}$, overlapping with another isomer), $2.25(\mathrm{~s}, 3 \mathrm{H}$, overlapping with another isomer), $2.16(\mathrm{~s}, 3 \mathrm{H})$.

13C NMR $\left(176 \mathrm{MHz}, \mathrm{CDCl}_{3}\right): \delta 142.1,131.2,127.9,127.4,122.6,115.2,20.5,17.5$.

2,6-dimethylaniline (6b) 
<smiles>Cc1cccc(C)c1N</smiles>

$\underline{1 \mathrm{H} \mathrm{NMR}}\left(700 \mathrm{MHz}, \mathrm{CDCl}_{3}\right): \delta 6.97(\mathrm{~d}, J=7.4 \mathrm{~Hz}, 2 \mathrm{H}), 6.67(\mathrm{t}, J=7.4 \mathrm{~Hz}, 1 \mathrm{H}), 3.50(\mathrm{~s}, 2 \mathrm{H}$, overlapping with another isomer), $2.20(\mathrm{~s}, 3 \mathrm{H})$.

13C NMR $\left(176 \mathrm{MHz}, \mathrm{CDCl}_{3}\right): \delta 142.8,128.3,121.8,118.1,17.6$.

3,5-dimethylaniline (6c)<smiles>Cc1cc(C)cc(N)c1</smiles>

1H NMR $\left(700 \mathrm{MHz}, \mathrm{CDCl}_{3}\right): \delta 6.44(\mathrm{~s}, 1 \mathrm{H}), 6.35(\mathrm{~s}, 2 \mathrm{H}), 3.50$ (s, 2H, overlapping with another isomer), 2.25 (s, 6H, overlapping with another isomer).

13C NMR $\left(176 \mathrm{MHz}, \mathrm{CDCl}_{3}\right): \delta 146.4,139.1,120.6,113.2,21.4$.

HRMS: (ESI) m/z [M+H]+ calcd. for $\mathrm{C}_{8} \mathrm{H}_{12} \mathrm{~N}+122.0964$; found 122.0963.

\section{3,4-dimethylaniline (7a), 2,3-dimethylaniline (7b)}<smiles>Cc1ccc(N)cc1C</smiles>

The general procedure was followed using ortho-xylene (106 $\mathrm{mg}, 1.0 \mathrm{mmol}$ ) and hydroxylamine hydrochloride (139 mg, $2.0 \mathrm{mmol}$ ) as starting materials. The crude mixture was purified by flash column chromatography (hexanes/EtOAc 5:1) to afford an inseparable mixture of the title compounds $(96.8 \mathrm{mg}, 80 \%)$ as a light yellow oil $(7 \mathbf{a}: 7 \mathbf{b}=2: 1)$.

3,4-dimethylaniline (7a)<smiles>Cc1ccc(N)cc1C</smiles>

$\underline{1 \mathrm{H} \mathrm{NMR}}\left(500 \mathrm{MHz}, \mathrm{CDCl}_{3}\right): \delta 6.96(\mathrm{~d}, J=7.9 \mathrm{~Hz}, 1 \mathrm{H}), 6.56(\mathrm{~d}, J=2.5 \mathrm{~Hz}, 1 \mathrm{H}), 6.50(\mathrm{dd}, J=7.9,2.5$ $\mathrm{Hz}, 1 \mathrm{H}), 3.54$ (br s, 2H), 2.24 (s, 3H), 2.21 (s, 3H).

13C NMR $\left(126 \mathrm{MHz}, \mathrm{CDCl}_{3}\right): \delta 144.2,137.4,130.4,126.6,117.0,112.7,19.9,18.8$.

2,3-dimethylaniline (7b) 
<smiles>Cc1cccc(N)c1C</smiles>

$\underline{1 \mathrm{H} \mathrm{NMR}}\left(500 \mathrm{MHz}, \mathrm{CDCl}_{3}\right): \delta 6.98(\mathrm{t}, J=7.7 \mathrm{~Hz}, 1 \mathrm{H}), 6.68(\mathrm{~d}, J=7.5 \mathrm{~Hz}, 1 \mathrm{H}), 6.61(\mathrm{~d}, J=7.9 \mathrm{~Hz}$, $1 \mathrm{H}), 3.54(\mathrm{br} \mathrm{s}, 2 \mathrm{H}), 2.32(\mathrm{~s}, 3 \mathrm{H}), 2.12(\mathrm{~s}, 3 \mathrm{H})$.

13C NMR $\left(126 \mathrm{MHz}, \mathrm{CDCl}_{3}\right): \delta$ 144.6, 137.2, 126.6, 121.0, 120.7, 113.3, 20.6, 12.8.

HRMS: (ESI) m/z [M+H]+ calcd. for $\mathrm{C}_{8} \mathrm{H}_{12} \mathrm{~N}+122.0964$; found 122.0963 .

The characterization data matched that of a commercial source.



The general procedure was followed using 1,2,3,4-tetrahydronaphthalene (132 $\mathrm{mg}, 1.0 \mathrm{mmol}$ ) and hydroxylamine hydrochloride $(139 \mathrm{mg}, 2.0 \mathrm{mmol}$ ) as starting materials. The crude product was purified by flash column chromatography (hexanes/EtOAc $5: 1$ ) to afford an inseparable mixture of the title compounds (96.8 $\mathrm{mg}, 66 \%)$ as a light yellow oil $(8 \mathbf{a}: \mathbf{8 b}=3: 1)$.

5,6,7,8-tetrahydronaphthalen-2-amine (8a)<smiles>Nc1ccc2c(c1)CCCC2</smiles>

$\underline{\text { 1H NMR }}\left(700 \mathrm{MHz}, \mathrm{CDCl}_{3}\right): \delta 6.87(\mathrm{~d}, J=8.2,1 \mathrm{H}), 6.49(\mathrm{~d}, J=8.1 \mathrm{~Hz}, 1 \mathrm{H}), 6.44(\mathrm{~s}, 1 \mathrm{H}), 3.52(\mathrm{br} \mathrm{s}$, $2 \mathrm{H}), 2.71-2.64(\mathrm{~m}, 4 \mathrm{H}), 1.81-1.70(\mathrm{~m}, 4 \mathrm{H})$.

13C NMR $\left(176 \mathrm{MHz}, \mathrm{CDCl}_{3}\right): \delta 143.9,138.0,130.0,127.5,115.6,113.3,29.6,28.7,23.7,23.4$.

5,6,7,8-tetrahydronaphthalen-1-amine $(\mathbf{8 b})$<smiles>Nc1cccc2c1CCCC2</smiles>

1H NMR $\left(700 \mathrm{MHz}, \mathrm{CDCl}_{3}\right): \delta 6.96(\mathrm{td}, J=7.7,3.4 \mathrm{~Hz}, 1 \mathrm{H}), 6.57(\mathrm{dt}, J=7.8,2.2 \mathrm{~Hz}, 1 \mathrm{H}), 6.53(\mathrm{~d}, J=$ $7.8 \mathrm{~Hz}, 1 \mathrm{H}), 3.52(\mathrm{br} \mathrm{s}, 2 \mathrm{H}), 2.75(\mathrm{~m}, 2 \mathrm{H}), 2.47(\mathrm{t}, J=6.5 \mathrm{~Hz}, 2 \mathrm{H}), 1.91-1.85(\mathrm{~m}, 2 \mathrm{H}), 1.77(\mathrm{~m}, 2 \mathrm{H})$.

13C NMR $\left(176 \mathrm{MHz}, \mathrm{CDCl}_{3}\right): \delta 144.4,138.2,126.0,121.9,119.7,112.2,30.0,24.2,22.9,22.9$.

HRMS: (ESI) m/z [M+H]+ calcd. for $\mathrm{C}_{10} \mathrm{H}_{14} \mathrm{~N}+148.1121$; found 148.1121 .

The characterization data matched that found in the literature (Chem. Eur. J. 2017, 23, 563-567). 
4-methoxybenzene-1,3-diamine (9)<smiles>COc1ccc(N)cc1N</smiles>

The general procedure was followed using 4-methoxyaniline (123 mg, $1.0 \mathrm{mmol}$ ) and hydroxylamine hydrochloride $(208.5 \mathrm{mg}, 3.0 \mathrm{mmol})$ as starting materials. The crude product was purified by flash column chromatography (hexanes/EtOAc 1:2 to 1:4) to afford 4-methoxybenzene-1,3-diamine as a grey solid that darkens rapidly in air (112 $\mathrm{mg}, 81 \%)$.

The ${ }^{1 \mathrm{H}} \mathrm{NMR},{ }^{13} \mathrm{C}$ NMR, and HRMS spectra were identical to compound $\mathbf{x x}$ reported above and matched that found in the literature (ACS Catal. 2016, 6, 8162-8165).

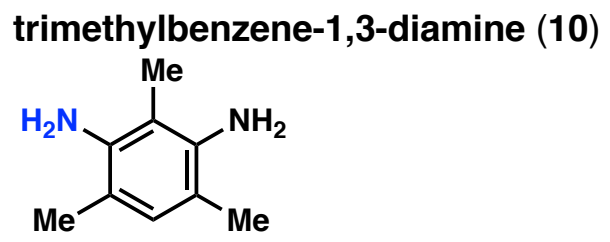

The general procedure was followed using 2,4,6-trimethylaniline (135 mg, $1.0 \mathrm{mmol}$ ) and hydroxylamine hydrochloride $(208.5 \mathrm{mg}, 3.0 \mathrm{mmol})$ as starting materials. The crude product was purified by flash column chromatography (hexanes/EtOAc 1:2 to 1:4) to afford the title compound as a yellow foam (94 $\mathrm{mg}, 62 \%)$.

$\underline{1 \mathrm{H} N M R}\left(500 \mathrm{MHz}, \mathrm{CDCl}_{3}\right): \delta 6.69(\mathrm{~s}, 1 \mathrm{H}), 3.46(\mathrm{~s}, 4 \mathrm{H}), 2.11(\mathrm{~s}, 6 \mathrm{H}), 2.03(\mathrm{~s}, 3 \mathrm{H})$.

13C NMR (126 MHz, $\left.\mathrm{CDCl}_{3}\right): \delta 141.1,129.4,112.6,107.0,17.3,11.0$.

HRMS: (ESI) m/z [M+H]+ calcd. for $\mathrm{C}_{9} \mathrm{H}_{15} \mathrm{~N}_{2}+151.1230$; found 151.1231 .

Melting Point: $52-53.5^{\circ} \mathrm{C}$

N-(4-methylthiophen-3-yl)acetamide (11a), $N$-(3-methylthiophen-2-yl)acetamide (11b)<smiles>CC(=O)Nc1cc(C)c[se]1</smiles>

A modified procedure was followed using 3-methylthiophene $(98 \mathrm{mg}, 1.0 \mathrm{mmol}$ ) and hydroxylamine hydrochloride (208 mg, $3.0 \mathrm{mmol}, 3.0$ equiv) as starting materials. The crude reaction was concentrated, and the resulting residue was dissolved in dichloromethane $(5.0 \mathrm{~mL})$ and treated with acetic anhydride (1.5 equiv) and triethylamine (2.0 equiv) at rt for $5 \mathrm{~h}$. Subsequently, the reaction was quenched with saturated sodium bicarbonate $(3 \mathrm{~mL})$ and extracted with dichloromethane $(3 \times 5 \mathrm{~mL})$, and the organic extracts were dried over magnesium sulfate. Purification by column chromatography (hexanes/EtOAc 2:1 to 1:1) afforded an inseparable mixture of the products as a cream colored solid (11a : 11b $1.33: 1,79 \mathrm{mg}, 51 \%$ ).

N-(4-methylthiophen-3-yl)acetamide (11a) 
$\overbrace{}^{S}$

1H NMR $\left(500 \mathrm{MHz}, \mathrm{DMSO}-d_{6}\right): \delta 10.98(\mathrm{~s}, 1 \mathrm{H}), 6.47(\mathrm{~s}, 1 \mathrm{H}), 6.44(\mathrm{~s}, 1 \mathrm{H}), 2.12(\mathrm{~s}, 3 \mathrm{H}), 2.03(\mathrm{~s}, 3 \mathrm{H})$.

${ }^{13} \mathrm{C}$ NMR $\left(126 \mathrm{MHz}, \mathrm{DMSO}-d_{6}\right): \delta 166.2,139.7,133.5,112.4,111.8,22.6,15.7$.

$N$-(3-methylthiophen-2-yl)acetamide (11b)<smiles>Cc1ccsc1NC(C)(F)Cl</smiles>

1H NMR (500 MHz, DMSO- $\left.d_{6}\right): \delta 10.06(\mathrm{~s}, 1 \mathrm{H}), 6.90(\mathrm{~d}, J=5.5 \mathrm{~Hz}, 1 \mathrm{H}), 6.69(\mathrm{~d}, J=5.5 \mathrm{~Hz}, 1 \mathrm{H}), 2.13$ $(\mathrm{s}, 3 \mathrm{H}), 2.09(\mathrm{~s}, 3 \mathrm{H})$.

$\underline{13 \mathrm{C} \mathrm{NMR}}\left(126 \mathrm{MHz}, \mathrm{DMSO}-d_{6}\right): \delta 167.1,133.3,127.1,121.2,117.0,22.5,12.9$.

HRMS: (ESI) m/z [M+H]+ calcd. for $\mathrm{C}_{7} \mathrm{H}_{10} \mathrm{NOS}+156.0478$; found 156.0478 .

The melting points were not taken as the products were isolated as an inseparable mixture by flash column chromatography.

\section{4-methoxy-3-methylaniline (12)}<smiles>COc1ccc(N)cc1C</smiles>

The general procedure was followed using 1-methoxy-2-methylbenzene (122 mg, $1.0 \mathrm{mmol}$ ) and hydroxylamine hydrochloride $(208.5 \mathrm{mg}, 3.0 \mathrm{mmol})$ as starting materials. The crude product was purified by flash column chromatography (hexanes/EtOAc $3: 1$ to $2: 1$ ) to afford the title compound as a yellow solid (109 $\mathrm{mg}, 80 \%)$.

1H NMR $\left(500 \mathrm{MHz}, \mathrm{CDCl}_{3}\right): \delta 6.67(\mathrm{~d}, J=8.4 \mathrm{~Hz}, 1 \mathrm{H}), 6.56-6.53(\mathrm{~m}, 1 \mathrm{H}), 6.51(\mathrm{dd}, J=8.4,2.9 \mathrm{~Hz}$, $1 \mathrm{H}), 3.76(\mathrm{~s}, 3 \mathrm{H}), 3.36(\mathrm{br} \mathrm{s}, 2 \mathrm{H}), 2.17(\mathrm{~s}, 3 \mathrm{H})$.

13C NMR $\left(126 \mathrm{MHz}, \mathrm{CDCl}_{3}\right): \delta 151.2,139.7,127.8,118.7,113.2,111.7,56.1,16.4$.

HRMS: (ESI) m/z [M+H]+ calcd. for $\mathrm{C}_{8} \mathrm{H}_{12} \mathrm{NO}+138.0913$; found 138.0913.

The characterization data matched that found in the literature (Chem. Eur. J. 2017, 23, 563-567).

2,3-dihydrobenzofuran-5-amine (13a), 2,3-dihydrobenzofuran-5,7-diamine (13b)<smiles>Nc1ccc2c(c1)CCO2</smiles> 
The general procedure was followed using dihydrobenzofuran (135 mg, $1.0 \mathrm{mmol}$ ) and hydroxylamine hydrochloride (173.7 $\mathrm{mg}, 2.5 \mathrm{mmol}$ ) as starting materials. The crude product was purified by flash column chromatography (hexanes/EtOAc 2:1 to 1:1) to afford 2,3-dihydrobenzofuran-5-amine as a yellow solid (74.3 mg, 55\%), followed by 2,3-dihydrobenzofuran-5,7-diamine as a brown solid (18.7 mg, 12\%).

2,3-dihydrobenzofuran-5-amine (13a)<smiles>Nc1ccc2c(c1)CCO2</smiles>

1H NMR (700 MHz, $\left.\mathrm{CDCl}_{3}\right): \delta 6.63-6.58(\mathrm{~m}, 2 \mathrm{H}), 6.46(\mathrm{dd}, J=8.5,2.3 \mathrm{~Hz}, 1 \mathrm{H}), 4.49(\mathrm{t}, J=8.6 \mathrm{~Hz}$, $2 \mathrm{H}), 3.39$ (br s, 2H), $3.13(\mathrm{t}, \mathrm{J}=8.6 \mathrm{~Hz}, 2 \mathrm{H})$.

${ }^{13} \mathrm{C} \mathrm{NMR}\left(176 \mathrm{MHz}, \mathrm{CDCl}_{3}\right): \delta$ 153.3, 140.0, 127.9, 114.8, 112.8, 109.4, 71.0, 30.4.

HRMS: $(E S I) \mathrm{m} / \mathrm{z}[\mathrm{M}+\mathrm{H}]+$ calcd. for $\mathrm{C}_{8} \mathrm{H}_{10} \mathrm{NO}+136.0757$; found 136.0756 .

The characterization data matched that found in the literature (ACS Catal. 2016, 6, 8162-8165).

2,3-dihydrobenzofuran-5,7-diamine (13b)<smiles>Nc1cc(N)c2c(c1)CCO2</smiles>

1H NMR (700 MHz, CDCl $): \delta 6.08-6.03(\mathrm{~m}, 1 \mathrm{H}), 5.97-5.93(\mathrm{~m}, 1 \mathrm{H}), 4.49(\mathrm{t}, J=8.6 \mathrm{~Hz}, 2 \mathrm{H}), 3.44(\mathrm{~s}$, $4 \mathrm{H}), 3.10(\mathrm{t}, J=8.6 \mathrm{~Hz}, 2 \mathrm{H})$.

${ }^{13} \mathrm{C} \mathrm{NMR}\left(176 \mathrm{MHz}, \mathrm{CDCl}_{3}\right): \delta 141.1,140.9,130.8,127.6,102.5,102.5,71.1,31.1$.

HRMS: (ESI) m/z [M+H]+ calcd. for $\mathrm{C}_{8} \mathrm{H}_{11} \mathrm{~N} 2 \mathrm{O}+151.0866$; found 151.0865 .

Melting Point: $114-116^{\circ} \mathrm{C}$ (decomposition)

\section{benzo[d][1,3]dioxol-5-amine (14)}<smiles>Nc1ccc2c(c1)OCO2</smiles>

The general procedure was followed using benzo[d][1,3]dioxole $(122 \mathrm{mg}, 1.0 \mathrm{mmol}$ ) and hydroxylamine hydrochloride $(208.5 \mathrm{mg}, 3.0 \mathrm{mmol})$ as starting materials and glacial acetic acid (instead of acetonitrile) as the solvent. The crude product was purified by flash column chromatography (hexanes/EtOAc $2: 1$ to $1: 1$ ) to afford the title compound as a yellow solid (78 mg, $57 \%)$.

1H NMR $\left(500 \mathrm{MHz}, \mathrm{CDCl}_{3}\right): \delta 6.62(\mathrm{~d}, J=8.2 \mathrm{~Hz}, 1 \mathrm{H}), 6.29(\mathrm{~d}, J=2.2 \mathrm{~Hz}, 1 \mathrm{H}), 6.13(\mathrm{dd}, J=8.2,2.3$ $\mathrm{Hz}, 1 \mathrm{H}), 5.86$ (s, 2H), 3.47 (br s, 2H).

$\underline{13 \mathrm{C} \mathrm{NMR}}\left(126 \mathrm{MHz}, \mathrm{CDCl}_{3}\right): \delta 148.3,141.5,140.5,108.7,107.0,100.8,98.2$. 
HRMS: (ESI) m/z [M+H] ${ }^{+}$calcd. for $\mathrm{C}_{7} \mathrm{H}_{8} \mathrm{NO}_{2}+138.0550$; found 138.0549 .

The characterization data matched that found in the literature (Chem. Eur. J. 2017, 23, 563-567).

6-methoxy-2,3-dihydro-1 $H$-inden-5-amine (15a), 5-methoxy-2,3-dihydro-1 $H$-inden-4-amine (15b)<smiles>COc1cc2c(cc1N)CCC2</smiles>

The general procedure was followed using 5-methoxy-2,3-dihydro-1 $\mathrm{H}$-indene (148.2 mg, $1.0 \mathrm{mmol})$ and hydroxylamine hydrochloride $(208.5 \mathrm{mg}, 3.0 \mathrm{mmol})$ as starting materials. The crude product was purified by flash column chromatography (hexanes/EtOAc $3: 1$ to $2: 1$ ) to afford a mixture of the title compounds as a wet, pale yellow solid $(73.3 \mathrm{mg}, 45 \%, 15 a: 15 b=2: 1)$.

6-methoxy-2,3-dihydro-1 $H$-inden-5-amine (15a)<smiles>COc1cc2c(cc1N)CCC2</smiles>

$\underline{1 H}$ NMR $\left(500 \mathrm{MHz}, \mathrm{CDCl}_{3}\right): \delta 6.71(\mathrm{~s}, 1 \mathrm{H}), 6.63(\mathrm{~s}, 1 \mathrm{H}), 3.83(\mathrm{~s}, 3 \mathrm{H}), 3.69(\mathrm{br} \mathrm{s}, 2 \mathrm{H}), 2.91-2.77(\mathrm{~m}$, 4H), 2.05 (pent, $J=7.4 \mathrm{~Hz}, 2 \mathrm{H}$ ).

13C NMR $\left(126 \mathrm{MHz}, \mathrm{CDCl}_{3}\right): \delta 146.5,136.2,134.6,134.0,111.3,107.2,55.9,32.9,32.7,25.9$.

5-methoxy-2,3-dihydro-1 $\mathrm{H}$-inden-4-amine (15b)<smiles>COc1ccc2c(c1N)CCC2</smiles>

1H NMR $\left(500 \mathrm{MHz}, \mathrm{CDCl}_{3}\right): \delta 6.66(\mathrm{~d}, J=8.0 \mathrm{~Hz}, 1 \mathrm{H}), 6.62(\mathrm{~d}, J=8.0 \mathrm{~Hz}, 1 \mathrm{H}), 3.84(\mathrm{~s}, 3 \mathrm{H}), 3.69(\mathrm{br}$ $\mathrm{s}, 2 \mathrm{H}), 2.87$ (t, $J=7.5 \mathrm{~Hz}, 2 \mathrm{H}), 2.74(\mathrm{t}, J=7.4 \mathrm{~Hz}, 2 \mathrm{H}), 2.12(\mathrm{p}, J=7.4 \mathrm{~Hz}, 2 \mathrm{H})$.

13C NMR $\left(126 \mathrm{MHz}, \mathrm{CDCl}_{3}\right): \delta 145.8,137.3,132.2,129.5,113.3,109.2,56.2,32.7,29.7,25.7$.

HRMS: (ESI) m/z [M+H]+ calcd. for $\mathrm{C}_{10} \mathrm{H}_{14} \mathrm{NO}+164.1070$; found 164.1071.

The characterization data matched that found in the literature (Chem. Eur. J. 2017, 23, 563-567).

The melting points were not taken as the products were isolated as an inseparable mixture by flash column chromatography.

$\mathrm{N}$-(2-aminophenyl)acetamide (16a), $\mathbf{N}$-(4-aminophenyl)acetamide (16b)<smiles>CC(=O)Nc1ccc(N)cc1</smiles> 
The general procedure was followed using $N$-phenylacetamide (135 mg, $1.0 \mathrm{mmol}$ ) and hydroxylamine hydrochloride $(208.5 \mathrm{mg}, 3.0 \mathrm{mmol}$ ) as starting materials. The crude products were purified by flash column chromatography (hexanes/EtOAc 1:1 to $1: 3$ ) to afford an inseparable mixture of the title compounds (16a : 16b $=2.44: 1)$ as a yellow solid (66 $\mathrm{mg}, 44 \%)$.

$N$-(2-aminophenyl)acetamide (16a)<smiles>CC(=O)Nc1ccc(N)cc1</smiles>

1H NMR: (500 MHz, DMSO- $\left.d_{6}\right): \delta 9.10(\mathrm{~s}, 1 \mathrm{H}), 7.14(\mathrm{dd}, J=7.8,1.5 \mathrm{~Hz}, 1 \mathrm{H}), 6.91-6.85(\mathrm{~m}, 1 \mathrm{H}), 6.70$ (dd, $J=7.9,1.4 \mathrm{~Hz}, 1 \mathrm{H}), 6.52$ (td, $J=7.6,1.5 \mathrm{~Hz}, 1 \mathrm{H}), 4.83(\mathrm{~s}, 2 \mathrm{H}), 2.03(\mathrm{~s}, 3 \mathrm{H})$.

$\underline{13 \mathrm{C} N M R}\left(126 \mathrm{MHz}, \mathrm{DMSO}-d_{6}\right): \delta 168.1,141.9,125.7,125.3,123.5,116.1,115.8,23.3$.

$N$-(4-aminophenyl)acetamide (16b)<smiles>CC(=O)Nc1ccccc1N</smiles>

1H NMR (500 MHz, DMSO- $\left.d_{6}\right): \delta 9.46(\mathrm{~s}, 1 \mathrm{H}), 7.18(\mathrm{~d}, J=8.7 \mathrm{~Hz}, 2 \mathrm{H}), 6.47(\mathrm{~d}, J=8.7 \mathrm{~Hz}, 2 \mathrm{H}), 4.80$ (br s, 2H), $1.94(\mathrm{~s}, 3 \mathrm{H})$.

13C NMR (126 MHz, DMSO- $\left.d_{6}\right): \delta 167.1,144.6,128.6,120.8,113.8,23.7$.

HRMS: (ESI) m/z [M+H]+ calcd. for $\mathrm{C}_{8} \mathrm{H}_{11} \mathrm{~N}_{2} \mathrm{O}+151.0866$; found 151.0866 .

The melting points were not taken as the products were isolated as an inseparable mixture by flash column chromatography.

The characterization data matched that found in the literature (ACS Catal. 2016, 6, 8162-8165).

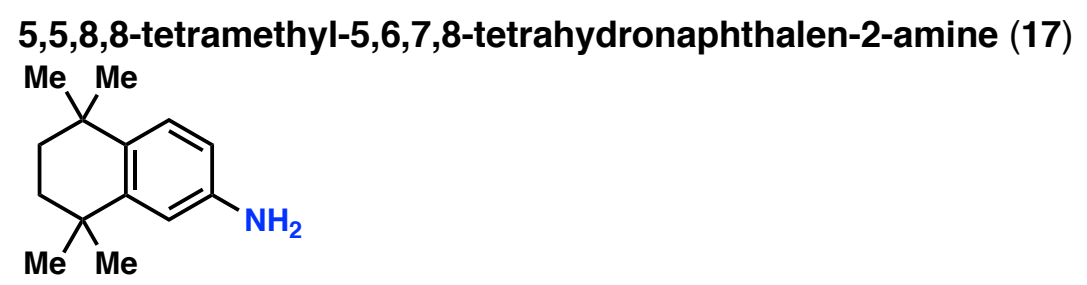

The general procedure was followed using 1,1,4,4-tetramethyl-1,2,3,4-tetrahydronaphthalene (188 $\mathrm{mg}, 1.0 \mathrm{mmol})$ and hydroxylamine hydrochloride $(208.5 \mathrm{mg}, 3.0 \mathrm{mmol})$ as starting materials. The crude product was purified by flash column chromatography (hexanes/EtOAc 9:1) to afford the title compound as a pale orange solid (172.6 $\mathrm{mg}, 85 \%)$.

$\underline{1 \mathrm{H} \mathrm{NMR}}\left(500 \mathrm{MHz}, \mathrm{CDCl}_{3}\right): \delta 7.10(\mathrm{dd}, J=8.3,1.6 \mathrm{~Hz}, 1 \mathrm{H}), 6.64(\mathrm{t}, J=2.0 \mathrm{~Hz}, 1 \mathrm{H}), 6.52(\mathrm{dd}, J=8.4$, $2.0 \mathrm{~Hz}, 1 \mathrm{H}), 3.50(\mathrm{br} \mathrm{s}, 2 \mathrm{H}), 1.65(\mathrm{~s}, 4 \mathrm{H}), 1.25(\mathrm{~s}, 6 \mathrm{H}), 1.24(\mathrm{~s}, 6 \mathrm{H})$. 
$13 \mathrm{C} \mathrm{NMR}\left(126 \mathrm{MHz}^{1} \mathrm{CDCl}_{3}\right): \delta 146.0,143.8,135.5,127.5,113.7,113.0,35.4(2 \mathrm{C}), 34.4,33.7,32.2$ (2C), $32.0(2 \mathrm{C})$.

HRMS: $(E S I) \mathrm{m} / \mathrm{z}[\mathrm{M}+\mathrm{H}]+$ calcd. for $\mathrm{C}_{14} \mathrm{H}_{22} \mathrm{~N}+204.1747$; found 204.1749 .

The characterization data matched that found in the literature. (Org. Lett. 2013, 15, 1378-1381).

\section{Scale-up procedure for compound 17:}

The scale-up procedure was adapted from the general procedure without further optimization.

1,1,4,4-tetramethyl-1,2,3,4-tetrahydronaphthalene $(1.88 \mathrm{~g}, 10.0 \mathrm{mmol})$ and hydroxylamine hydrochloride $(2.08 \mathrm{mg}, 30.0 \mathrm{mmol})$ were suspended in dry acetonitrile $(20 \mathrm{~mL})$. The resulting solution was immersed in a rt $\left(25^{\circ} \mathrm{C}\right)$ water bath, and concentrated sulfuric acid was added slowly $(5.33 \mathrm{~mL}$, $100 \mathrm{mmol})$. The water bath was removed, and the reaction was allowed to warm to $\mathrm{rt}\left(25^{\circ} \mathrm{C}\right)$. The reaction flask was then sealed with a septum, and the headspace was flushed with nitrogen for 5 min. $\mathrm{TiCl}_{3}(12 \%$ in aqueous $\mathrm{HCl}$ ) was added via a syringe pump (addition rate of $20 \mathrm{~mL}$ per hour) until the purple color of the $\mathrm{TiCl}_{3}$ solution persisted for more than $10 \mathrm{~min}$. During the addition, the reaction temperature was routinely checked, and the addition rate was adjusted to ensure the temperature was kept below $30^{\circ} \mathrm{C}$. The reaction was stirred for an additional 10 min before saturated aqueous sodium citrate $(30 \mathrm{~mL})$ was added. Sodium hydroxide $(20 \%)$ was then added slowly until the reaction turned basic. The reaction mixture was subsequently extracted with ethyl acetate $(5 \times 20 \mathrm{~mL})$. The combined organic phases were then dried over magnesium sulfate and purified by column chromatography over silica. The product was isolated as a pale orange solid $(1.65 \mathrm{~g}, 81.5 \%)$ together with a smaller fraction of 5,5,8,8-tetramethyl-5,6,7,8-tetrahydronaphthalene-2,3-diamine, 17b (196 mg, 9\%) which was isolated as a pale yellow solid that turned brown after prolonged storage on the benchtop.

\section{5,5,8,8-tetramethyl-5,6,7,8-tetrahydronaphthalene-2,3-diamine (17b)}<smiles>CC1(C)CCC(C)(C)c2cc(N)c(N)cc21</smiles>

1H NMR (700 MHz, $\left.\mathrm{CDCl}_{3}\right): \delta 6.63(\mathrm{~s}, 2 \mathrm{H}), 3.29(\mathrm{~s}, 4 \mathrm{H}), 1.63(\mathrm{~s}, 4 \mathrm{H}), 1.23(\mathrm{~s}, 12 \mathrm{H})$.

13C NMR (176 MHz, CDCl $)$ : $\delta 137.0$ (2C), 132.8 (2C), 114.7 (2), 35.5 (2C), 33.8 (2C), 32.2 (4C).

HRMS: (ESI) m/z [M+H]+ calcd. for $\mathrm{C}_{14} \mathrm{H}_{23} \mathrm{~N}_{2} \mathrm{O}+219.1856$; found 219.1855.

Melting Point: $111-113^{\circ} \mathrm{C}$ (decomposition)

3-bromo-4-methoxyaniline (18)<smiles>COc1ccc(N)cc1Br</smiles> 
The general procedure was followed using 2-bromoanisole (186 mg, $1.0 \mathrm{mmol}$ ) and hydroxylamine hydrochloride (208.5 mg, $3.0 \mathrm{mmol}$ ) as starting materials and glacial acetic acid (instead of acetonitrile) as the solvent. The crude product was purified by flash column chromatography (hexanes/EtOAc 5:1) to afford the title compound as a yellow oil (141 mg, 70\%).

1H NMR $\left(500 \mathrm{MHz}, \mathrm{CDCl}_{3}\right): \delta 6.93(\mathrm{~d}, J=2.7 \mathrm{~Hz}, 1 \mathrm{H}), 6.74(\mathrm{~d}, J=8.6 \mathrm{~Hz}, 1 \mathrm{H}), 6.60(\mathrm{dd}, J=8.6,2.8$ $\mathrm{Hz}, 1 \mathrm{H}), 3.81(\mathrm{~s}, 3 \mathrm{H}), 3.46(\mathrm{br} \mathrm{s}, 2 \mathrm{H})$.

13C NMR $\left(126 \mathrm{MHz}, \mathrm{CDCl}_{3}\right): \delta$ 149.2, 141.2, 120.4, 115.2, 113.8, 112.5, 57.1.

HRMS: (ESI) m/z [M+H]+ calcd. for $\mathrm{C}_{7} \mathrm{H}_{9} \mathrm{BrNO}+201.9862$; found 201.9863.

The characterization data matched that found in the literature (Chem. Eur. J. 2017, 23, 563-567).

2-chloro-3,5-dimethoxyaniline (19a), 5-chloro-2,4-dimethoxyaniline (19b)<smiles>COc1cc(Cl)c(OC)cc1N</smiles>

The general procedure was followed using 1-chloro-2,4-dimethoxybenzene (172 $\mathrm{mg}, 1.0 \mathrm{mmol}$ ) and hydroxylamine hydrochloride $(139 \mathrm{mg}, 2.0 \mathrm{mmol})$ as starting materials. The crude product mixture was purified by flash column chromatography (hexanes/EtOAc $4: 1$ to $3: 2$ to $2: 3$ ) to afford a yellow oil (19a, $24 \mathrm{mg}, 13 \%)$, followed by a low-melting pale yellow solid (19b, $89 \mathrm{mg}, 47 \%)$.

\section{2-chloro-3,5-dimethoxyaniline (19a)}<smiles>COc1cc(N)c(Cl)c(OC)c1</smiles>

1H NMR $\left(700 \mathrm{MHz}, \mathrm{CDCl}_{3}\right): \delta 6.72(\mathrm{~s}, 1 \mathrm{H}), 6.50(\mathrm{~s}, 1 \mathrm{H}), 3.84(\mathrm{~s}, 3 \mathrm{H}), 3.84(\mathrm{~s}, 3 \mathrm{H}), 3.57(\mathrm{~s}, 2 \mathrm{H})$.

13C NMR $\left(176 \mathrm{MHz}, \mathrm{CDCl}_{3}\right): \delta 147.86,146.68,130.81,116.34,114.12,98.97,57.63,56.01$.

HRMS (ESI) m/z [M+H]+ calcd. for $\mathrm{C}_{8} \mathrm{H}_{11} \mathrm{CINO}_{2}+188.0473$; found 188.0473.

\section{5-chloro-2,4-dimethoxyaniline (19b)}<smiles>COc1cc(OC)c(Cl)cc1N</smiles>

1H NMR: $\left(500 \mathrm{MHz}, \mathrm{CDCl}_{3}\right): \delta 6.46(\mathrm{~s}, 1 \mathrm{H}), 6.21(\mathrm{~s}, 1 \mathrm{H}), 3.79(\mathrm{~s}, 6 \mathrm{H}), 3.47(\mathrm{~s}, 2 \mathrm{H})$.

13C NMR: (126 MHz, $\left.\mathrm{CDCl}_{3}\right): \delta$ 140.23, 130.41, 104.43, 99.73, 57.02.

HRMS: (ESI) m/z [M+H]+ calcd. for $\mathrm{C}_{8} \mathrm{H}_{11} \mathrm{CINO}_{2}+188.0473$; found 188.0473.

Melting Point: $34-36^{\circ} \mathrm{C}$ 
5-amino-2-methoxybenzamide (20)<smiles>COc1ccc(N)cc1C(N)=O</smiles>

The general procedure was followed using 2-methoxybenzamide (151 mg, $1.0 \mathrm{mmol}$ ) and hydroxylamine hydrochloride $(208.5 \mathrm{mg}, 3.0 \mathrm{mmol})$ as starting materials. The crude product was purified by flash column chromatography (hexanes/EtOAc 0:1) to afford the title compound as a white solid (157 mg, 95\%).

1H NMR (700 MHz, $\left.\mathrm{CDCl}_{3}\right): \delta 7.84(\mathrm{br} \mathrm{s}, 1 \mathrm{H}), 7.55(\mathrm{~d}, J=2.7 \mathrm{~Hz}, 1 \mathrm{H}), 6.86-6.73(\mathrm{~m}, 2 \mathrm{H}), 5.90$ (br s, $1 \mathrm{H}), 3.89(\mathrm{~d}, J=1.3 \mathrm{~Hz}, 3 \mathrm{H}), 3.56(\mathrm{br} \mathrm{s}, 2 \mathrm{H})$.

$\underline{13 \mathrm{C} \mathrm{NMR}}\left(176 \mathrm{MHz}, \mathrm{CDCl}_{3}\right): \delta 167.2,151.2,140.5,121.3,120.0,118.8,113.1,56.6$.

HRMS: (ESI) $\mathrm{m} / \mathrm{z}[\mathrm{M}+\mathrm{H}]+$ calcd. for $\mathrm{C}_{8} \mathrm{H}_{11} \mathrm{~N}_{2} \mathrm{O}+167.0815$; found 167.0815 .

Melting Point: $73-76^{\circ} \mathrm{C}$

methyl 5-amino-2-methoxybenzoate (21)<smiles>COC(=O)c1cc(N)ccc1OC</smiles>

The general procedure was followed using methyl 2-methoxybenzoate (166 mg, $1.0 \mathrm{mmol}$ ) and hydroxylamine hydrochloride $(208.5 \mathrm{mg}, 3.0 \mathrm{mmol})$ as starting materials. The crude product was purified by flash column chromatography (hexanes/EtOAc 4:1) to afford the title compound as a pale yellow oil (128 mg, 71\%).

$\underline{1 \mathrm{H} \mathrm{NMR}}\left(500 \mathrm{MHz}, \mathrm{CDCl}_{3}\right): \delta 7.14(\mathrm{dd}, J=2.1,1.2 \mathrm{~Hz}, 1 \mathrm{H}), 6.85-6.78(\mathrm{~m}, 2 \mathrm{H}), 3.87(\mathrm{~s}, 3 \mathrm{H}), 3.82(\mathrm{~s}$, $3 \mathrm{H}), 3.50(\mathrm{~s}, 2 \mathrm{H})$.

13C NMR $\left(176 \mathrm{MHz}, \mathrm{CDCl}_{3}\right): \delta 166.9,152.5,139.7,120.6,120.4,118.2,114.2,56.9,52.2$.

HRMS: (ESI) m/z [M+H]+ calcd. for $\mathrm{C}_{9} \mathrm{H}_{12} \mathrm{NO}_{3}+182.0812$; found 182.0813 .

The characterization data matched that found in the literature (Adv. Synth. Catal. 2010, 352, 24632473).

\section{6-methoxy-2-methylbenzo[d]thiazol-7-amine $\quad(22 a), \quad$ 6-methoxy-2-methylbenzo[d]thiazol-5- amine (22b)}<smiles>CO[C@H]1CC=c2sc(C)nc2=C1N</smiles>

The general procedure was followed using 6-methoxyl-2-methylbenzothiazole $(89.5 \mathrm{mg}, 0.50 \mathrm{mmol})$, hydroxylamine hydrochloride $(105 \mathrm{mg}, 1.5 \mathrm{mmol})$ as starting materials and acetonitrile $(1 \mathrm{~mL})$ as the solvent. The crude product was purified by flash column chromatography (hexanes/EtOAc $5: 1$ to $2: 3$ ) 
to afford 6-methoxy-2-methylbenzo[ $d$ ]thiazol-7-amine as a pale grey solid (39.2 mg, 40\%), followed by 6-methoxy-2-methylbenzo[d]thiazol-5-amine as a tan solid $(25.5 \mathrm{mg}, 26 \%)$.

6-methoxy-2-methylbenzo[d]thiazol-7-amine (22a)<smiles>COc1ccc2sc(C)nc2c1N</smiles>

1H NMR $\left(700 \mathrm{MHz}, \mathrm{CDCl}_{3}\right): \delta 7.75(\mathrm{~d}, J=8.6 \mathrm{~Hz}, 1 \mathrm{H}), 7.38(\mathrm{~d}, J=8.7 \mathrm{~Hz}, 1 \mathrm{H}), 4.30(\mathrm{br} \mathrm{s}, 2 \mathrm{H}), 4.28$ $(\mathrm{s}, 3 \mathrm{H}), 3.16(\mathrm{~s}, 3 \mathrm{H})$.

${ }^{13} \mathrm{C} \mathrm{NMR}\left(176 \mathrm{MHz}, \mathrm{CDCl}_{3}\right): \delta 164.2,148.8,143.7,129.6,122.9,112.1,110.8,56.7,20.3$.

HRMS: (ESI) m/z [M+H]+ calcd. for $\mathrm{C}_{9} \mathrm{H}_{11} \mathrm{~N}_{2} \mathrm{OS}+195.0587$; found 195.0587.

Melting Point: $120-122^{\circ} \mathrm{C}$

6-methoxy-2-methylbenzo[d]thiazol-5-amine (22b)<smiles>COc1cc2nc(C)sc2cc1N</smiles>

1H NMR $\left(700 \mathrm{MHz}, \mathrm{CDCl}_{3}\right) \delta 7.25(\mathrm{~s}, 1 \mathrm{H}), 7.13(\mathrm{~s}, 1 \mathrm{H}), 3.95$ (br s, 2H), 3.90 (s, 3H), $2.75(\mathrm{~s}, 3 \mathrm{H})$.

${ }^{13} \mathrm{C} \mathrm{NMR}\left(176 \mathrm{MHz}, \mathrm{CDCl}_{3}\right) \delta 164.5,148.2,146.9,136.2,125.2,107.0,101.9,56.0,20.0$.

HRMS: (ESI) m/z [M+H]+ calcd. for $\mathrm{C}_{9} \mathrm{H}_{11} \mathrm{~N}_{2} \mathrm{OS}+195.0587$; found 195.0585.

Melting Point: $113-115^{\circ} \mathrm{C}$

5,7-dimethylbenzo[d]thiazole-2,6-diamine (23b)<smiles>Cc1cc2nc(N)sc2c(C)c1N</smiles>

The general procedure was followed using 5,7-dimethylbenzo[d]thiazol-2-amine (89 $\mathrm{mg}, 0.50 \mathrm{mmol})$ and hydroxylamine hydrochloride $(105 \mathrm{mg}, 1.5 \mathrm{mmol})$ as starting materials and acetonitrile $(1 \mathrm{~mL})$ as the solvent. The crude products were purified by flash column chromatography (hexanes/EtOAc 1:1 to $0: 1$ ) to afford a mixture of the title compounds as a pale yellow powder (45 $\mathrm{mg}, 50 \%)$. A small sample was purified via preparative TLC (100\% EtOAc as eluent) to separate the two isomers for further characterization.

\section{5,7-dimethylbenzo[d]thiazole-2,6-diamine (23a)}


<smiles>Cc1cc2nc(N)sc2c(C)c1N</smiles>

1H NMR (700 MHz, CD $\left.{ }_{3} \mathrm{OD}\right): \delta 6.62(\mathrm{~s}, 1 \mathrm{H}), 2.25(\mathrm{~s}, 3 \mathrm{H}), 2.20(\mathrm{~s}, 3 \mathrm{H}), \mathrm{NH}_{2}$ protons not observed due to rapid exchange.

13C NMR (176 MHz, CD $\left.{ }_{3} \mathrm{OD}\right): \delta 167.8,141.3,134.1,129.4,125.7,121.1,121.0,20.4,17.1$.

HRMS: (ESI) m/z [M+H]+ calcd. for $\mathrm{C}_{9} \mathrm{H}_{12} \mathrm{~N}_{3} \mathrm{~S}+194.0746$; found 194.0745.

\section{5,7-dimethylbenzo[d]thiazole-2,4-diamine (23b)}<smiles>Cc1cc(C)c2sc(N)nc2c1N</smiles>

1H NMR $\left(700 \mathrm{MHz}, \mathrm{CD}_{3} \mathrm{OD}\right): \delta 7.00(\mathrm{~s}, 1 \mathrm{H}), 2.25(\mathrm{~s}, 3 \mathrm{H}), 2.23(\mathrm{~s}, 3 \mathrm{H}), \mathrm{NH}_{2}$ protons not observed due to rapid exchange.

$13 \mathrm{C} \mathrm{NMR}\left(176 \mathrm{MHz}, \mathrm{CD}_{3} \mathrm{OD}\right): \delta 165.4,143.4,137.7,129.6,121.8,116.8,114.2,16.9,15.7$.

HRMS: (ESI) m/z [M+H]+ calcd. for $\mathrm{C}_{9} \mathrm{H}_{12} \mathrm{~N}_{3} \mathrm{~S}+194.0746$; found 194.0745.

$\mathrm{N}$-(3-amino-2,6-dimethylphenyl)-2-(diethylamino)acetamide (24a), $\mathrm{N}$-(4-amino-2,6dimethylphenyl)-2-(diethylamino)acetamide (24b)<smiles>CCN(CC)CC(=O)Nc1c(C)ccc(N)c1C</smiles>

The general procedure was followed using lidocaine (234 $\mathrm{mg}, 1.0 \mathrm{mmol}$ ) and hydroxylamine hydrochloride $(208 \mathrm{mg}, 3.0 \mathrm{mmol})$ as starting materials. The crude product was purified by flash column chromatography (hexanes/EtOAc $1: 1$ to $0: 1$ ) to afford an inseparable mixture of the title compounds (5.56:1) as a cream colored powder (199 $\mathrm{mg}, 80 \%)$.

$\mathrm{N}$-(3-amino-2,6-dimethylphenyl)-2-(diethylamino)acetamide (24a)<smiles>CCN(CC)CC(=O)Nc1c(C)ccc(N)c1C</smiles>

1H NMR $\left(700 \mathrm{MHz}, \mathrm{CDCl}_{3}\right): \delta 8.90(\mathrm{br} \mathrm{s}, 1 \mathrm{H}), 6.90(\mathrm{~d}, J=8.1 \mathrm{~Hz}, 1 \mathrm{H}), 6.58(\mathrm{~d}, J=8.0 \mathrm{~Hz}, 1 \mathrm{H}), 3.53$ (br s, 2H), $3.22(\mathrm{~s}, 2 \mathrm{H}), 2.69$ (q, J=7.1 Hz, 4H), $2.13(\mathrm{~d}, J=2.2 \mathrm{~Hz}, 3 \mathrm{H}), 2.02(\mathrm{~s}, 3 \mathrm{H}), 1.13(\mathrm{t}, J=7.2$ $\mathrm{Hz}, 6 \mathrm{H})$.

13C NMR $\left(176 \mathrm{MHz}, \mathrm{CDCl}_{3}\right): \delta 170.6,143.5,134.3,128.9,124.8,120.3,114.4,57.6,49.1,18.24,12.8$, 12.60 . 
$\mathrm{N}$-(4-amino-2,6-dimethylphenyl)-2-(diethylamino)acetamide (24b)<smiles>CCN(CC)CC(=O)Nc1c(C)cc(N)cc1C</smiles>

1H NMR (700 MHz, CDCl $)$ : $\delta 8.70(\mathrm{br} \mathrm{s}, 1 \mathrm{H}), 6.41$ (s, 2H), $3.52(\mathrm{br} \mathrm{s}, 2 \mathrm{H}) 3.19(\mathrm{~s}, 2 \mathrm{H}), 2.66$ (t, J = 7.1 $\mathrm{Hz}, 4 \mathrm{H}), 2.12(\mathrm{~s}, 6 \mathrm{H}), 1.12(\mathrm{t}, J=7.1 \mathrm{~Hz}, 6 \mathrm{H})$.

13C NMR $\left(176 \mathrm{MHz}, \mathrm{CDCl}_{3}\right): \delta$ 170.99, 145.4, 136.4, 125.1, 114.9, 57.6, 49.1, 18.7, 12.8.

HRMS: (ESI) m/z [M+H]+ calcd. for $\mathrm{C}_{14} \mathrm{H}_{24} \mathrm{~N}_{3} \mathrm{O}+250.1914$; found 250.1916.

The melting points were not taken as the products were isolated as an inseparable mixture by flash column chromatography.



The general procedure was followed using dextromethorphan $(135.6 \mathrm{mg}, 0.5 \mathrm{mmol})$, hydroxylamine hydrochloride (104 mg, $1.5 \mathrm{mmol}$ ) as starting materials and acetonitrile $(1 \mathrm{~mL})$ as the solvent. Dextromethorphan was used as a free base, as described in the literature. The crude product was purified by flash column chromatography (EtOAc/MeOH/Et ${ }_{3} \mathrm{~N}$ 1:0:0.05 to 19:1:0.5) to afford the title compound as a pale yellow gum (93 $\mathrm{mg}, 65 \%)$.

1H NMR $\left(500 \mathrm{MHz}, \mathrm{CDCl}_{3}\right): \delta 6.61(\mathrm{~s}, 1 \mathrm{H}), 6.46(\mathrm{~s}, 1 \mathrm{H}), 3.80(\mathrm{~s}, 3 \mathrm{H}), 3.65(\mathrm{br} \mathrm{s}, 2 \mathrm{H}), 2.86(\mathrm{~d}, J=18.2$ $\mathrm{Hz}, 1 \mathrm{H}), 2.79(\mathrm{dd}, J=5.8,3.2 \mathrm{~Hz}, 1 \mathrm{H}), 2.58-2.52(\mathrm{~m}, 1 \mathrm{H}), 2.45$ (ddd, $J=11.9,4.8,1.8 \mathrm{~Hz}, 1 \mathrm{H}), 2.39$ (s, 3H), 2.31-2.24 (m, 1H), $2.13(\mathrm{td}, J=12.3,3.4 \mathrm{~Hz}, 1 \mathrm{H}), 1.80(\mathrm{dt}, J=12.8,3.2 \mathrm{~Hz}, 1 \mathrm{H}), 1.71$ (td, $J$ $=12.7,4.8 \mathrm{~Hz}, 1 \mathrm{H}), 1.65-1.59(\mathrm{~m}, 1 \mathrm{H}), 1.49(\mathrm{dt}, J=8.5,2.7 \mathrm{~Hz}, 1 \mathrm{H}), 1.40-1.26(\mathrm{~m}, 5 \mathrm{H}), 1.03(\mathrm{t}, J=$ $7.2 \mathrm{~Hz}, 1 \mathrm{H})$.

$13 \mathrm{C} \mathrm{NMR}\left(126 \mathrm{MHz}, \mathrm{CDCl}_{3}\right): \delta$ 146.6, 133.8, 130.0, 129.8, 114.3, 107.4, 58.3, 55.8, 47.5, 45.4, 42.8, $42.0,36.8,36.7,26.7,26.7,23.7,22.3$.

HRMS: (ESI) m/z [M+H]+ calcd. for $\mathrm{C}_{18} \mathrm{H}_{27} \mathrm{~N}_{2} \mathrm{O}+287.2118$; found 287.2117.

The characterization data matched that found in the literature (ACS Catal. 2016, 6, 8162-8165).

amino-julolidine (26)<smiles>Nc1ccc2c3c1CCCN3CCC2</smiles> 
Procedure for julolidine hydrochloride: Julolidine $\left(0.5 \mathrm{~g}, 2.9 \mathrm{mmol}\right.$, Alfa Aesar) was suspended in $\mathrm{Et}_{2} \mathrm{O}$ $(10 \mathrm{~mL})$ and passed through a pipette silica plug (2 in). The silica plug was washed with more $\mathrm{Et}_{2} \mathrm{O}$ $(10 \mathrm{~mL})$ to provide a pale yellow solution. Anhydrous $\mathrm{HCl}$ in $\mathrm{Et}_{2} \mathrm{O}(2 \mathrm{M}, 1.5 \mathrm{~mL}, 3.0 \mathrm{mmol})$ was then added dropwise, resulting in the precipitation of the julolidine $\mathrm{HCl}$ salt. The suspension was allowed to stand for 10 min, after which the precipitated solids were filtered and dried.

Procedure for amino-julolidine: The general procedure was followed using julolidine hydrochloride (104.5 mg, $0.5 \mathrm{mmol}$ ) and hydroxylamine hydrochloride (104 mg, $1.5 \mathrm{mmol}$ ) as starting materials and glacial acetic acid $(1 \mathrm{~mL})$ as the solvent. The crude product was purified by flash column chromatography (hexanes/EtOAc $4: 1$ ) to afford the title compound as a brown oil (52 mg, 55\%).

1H NMR $\left(700 \mathrm{MHz}, \mathrm{CDCl}_{3}\right): \delta 6.65(\mathrm{dt}, J=7.9,0.9 \mathrm{~Hz}, 1 \mathrm{H}), 6.04(\mathrm{~d}, J=7.9 \mathrm{~Hz}, 1 \mathrm{H}), 3.43(\mathrm{br} \mathrm{s}, 2 \mathrm{H})$, 3.10-3.02 (m, 4H), $2.69(\mathrm{t}, J=6.6 \mathrm{~Hz}, 2 \mathrm{H}), 2.49(\mathrm{t}, \mathrm{J}=6.8 \mathrm{~Hz}, 2 \mathrm{H}), 2.08-2.00(\mathrm{~m}, 2 \mathrm{H})$, 2.00-1.92 (m, $2 \mathrm{H})$.

$13 \mathrm{C} \mathrm{NMR}\left(176 \mathrm{MHz}, \mathrm{CDCl}_{3}\right): \delta$ 143.9, 142.6, 127.2, 113.0, 107.1, 104.4, 50.7, 49.6, 27.4, 22.8, 22.4, 22.1.

HRMS: (ESI) m/z [M+H]+ calcd. for $\mathrm{C}_{12} \mathrm{H}_{17} \mathrm{~N}_{2}+189.1386$; found 189.1387 .

The characterization data matched that found in the literature (Synlett 2017, 28, 1652-1656.)<smiles>Nc1cc2c(cc1OCCCCN1CCN(c3cccc(Cl)c3Cl)CC1)NC(=O)CC2</smiles>

The general procedure was followed using azipiprazole (89 $\mathrm{mg}, 0.2 \mathrm{mmol}$ ) and hydroxylamine hydrochloride (41 mg, $0.6 \mathrm{mmol})$ as starting materials, sulfuric acid $(2 \mathrm{mmol})$ as an additive, and acetonitrile $(0.4 \mathrm{~mL})$ as the solvent. The crude product was purified by preparative TLC $\left(\mathrm{CH}_{2} \mathrm{Cl}_{2} / \mathrm{MeOH} / \mathrm{Et}_{3} \mathrm{~N}\right.$ 19:1:0.5) to yield $27 \mathrm{a}$ as an off-white powder (contaminated with unreacted starting material) and $\mathbf{2 7 b}$ as a yellow wax (60 $\mathrm{mg}, 65 \%$ ). 27 a can be further purified via careful preparative TLC (EtOAc/MeOH/Et ${ }_{3} \mathrm{~N}$ 19.5:0.5:0.5) to obtain a pure, clear film $(9.2 \mathrm{mg}, 10 \%)$.

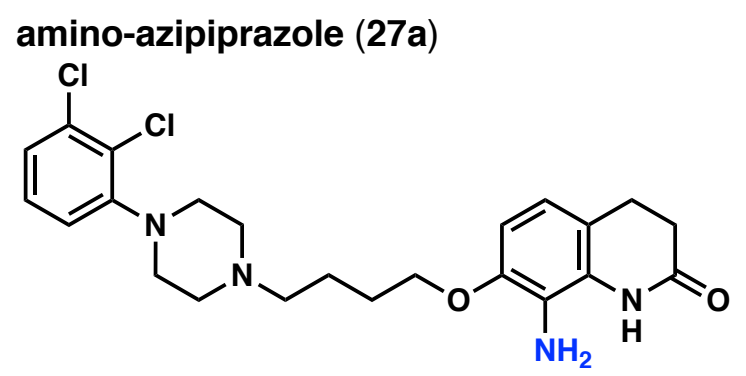

1H NMR (700 MHz, CDCl 3 ): $\delta 8.65(\mathrm{br} \mathrm{s}, 1 \mathrm{H}), 7.17-7.09(\mathrm{~m}, 2 \mathrm{H}), 6.96(\mathrm{dd}, J=7.3,2.3 \mathrm{~Hz}, 1 \mathrm{H}), 6.57$ $(\mathrm{d}, J=8.2 \mathrm{~Hz}, 1 \mathrm{H}), 6.49(\mathrm{~d}, J=8.2 \mathrm{~Hz}, 1 \mathrm{H}), 4.02(\mathrm{t}, J=6.3 \mathrm{~Hz}, 2 \mathrm{H}), 3.79(\mathrm{br} \mathrm{s}, 2 \mathrm{H}), 3.08(\mathrm{br} \mathrm{s}, 4 \mathrm{H})$, 2.88 (dd, $J=8.4,6.3 \mathrm{~Hz}, 2 \mathrm{H}), 2.65(\mathrm{br} \mathrm{s}, 4 \mathrm{H}), 2.61-2.56(\mathrm{~m}, 2 \mathrm{H}), 2.53-2.45(\mathrm{~m}, 2 \mathrm{H}), 1.93-1.81(\mathrm{~m}$, $2 \mathrm{H}), 1.78-1.68(\mathrm{~m}, 2 \mathrm{H})$. 
$13 \mathrm{C} \mathrm{NMR}\left(176 \mathrm{MHz}, \mathrm{CDCl}_{3}\right): \delta 172.3,151.4,147.0,134.2,127.7,127.6,126.1,124.7,123.4,118.7$, $117.5,117.4,106.2,68.6,58.4,53.5,51.5,31.4,27.6,25.4,23.7$.

HRMS: (ESI) m/z [M+H]+ calcd. for $\mathrm{C}_{23} \mathrm{H}_{29} \mathrm{Cl}_{2} \mathrm{~N}_{4} \mathrm{O}_{2}+463.1662$; found 463.1661.

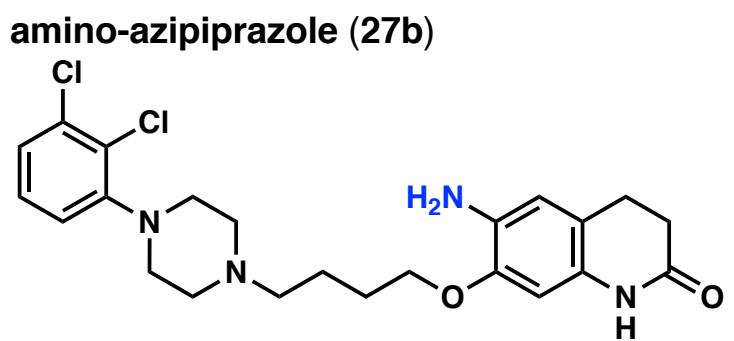

1H NMR $\left(700 \mathrm{MHz}, \mathrm{CDCl}_{3}\right): \delta 8.33(\mathrm{br} \mathrm{s}, 1 \mathrm{H}), 7.18-7.08(\mathrm{~m}, 2 \mathrm{H}), 6.95(\mathrm{dd}, J=6.8,2.8 \mathrm{~Hz}, 1 \mathrm{H}), 6.53$ $(\mathrm{s}, 1 \mathrm{H}), 6.27(\mathrm{~s}, 1 \mathrm{H}), 3.99(\mathrm{t}, J=6.3 \mathrm{~Hz}, 2 \mathrm{H}), 3.62(\mathrm{br} \mathrm{s}, 2 \mathrm{H}), 3.07$ (br s, $4 \mathrm{H}), 2.81$ (dd, $J=8.5,6.6 \mathrm{~Hz}$, 2H), 2.65 (br s, 4H), 2.57 (dd, J = 8.6, $6.5 \mathrm{~Hz}, 2 \mathrm{H}), 2.52-2.44(\mathrm{~m}, 2 \mathrm{H}), 1.91-1.81(\mathrm{~m}, 2 \mathrm{H}), 1.77-1.67$ $(\mathrm{m}, 2 \mathrm{H})$.

$13 \mathrm{C} \mathrm{NMR}\left(176 \mathrm{MHz}, \mathrm{CDCl}_{3}\right): \delta 171.7,151.4,146.0,134.1,131.8,129.0,127.6,127.6,124.7,118.7$, $115.9,114.9,100.5,68.6,58.3,53.4,51.4,31.3,27.5,25.0,23.6$.

HRMS: (ESI) m/z [M+H]+ calcd. for $\mathrm{C}_{23} \mathrm{H}_{29} \mathrm{Cl}_{2} \mathrm{~N}_{4} \mathrm{O}_{2}+463.1662$; found 463.1662. 


\section{Kinetic Isotope Experiments}
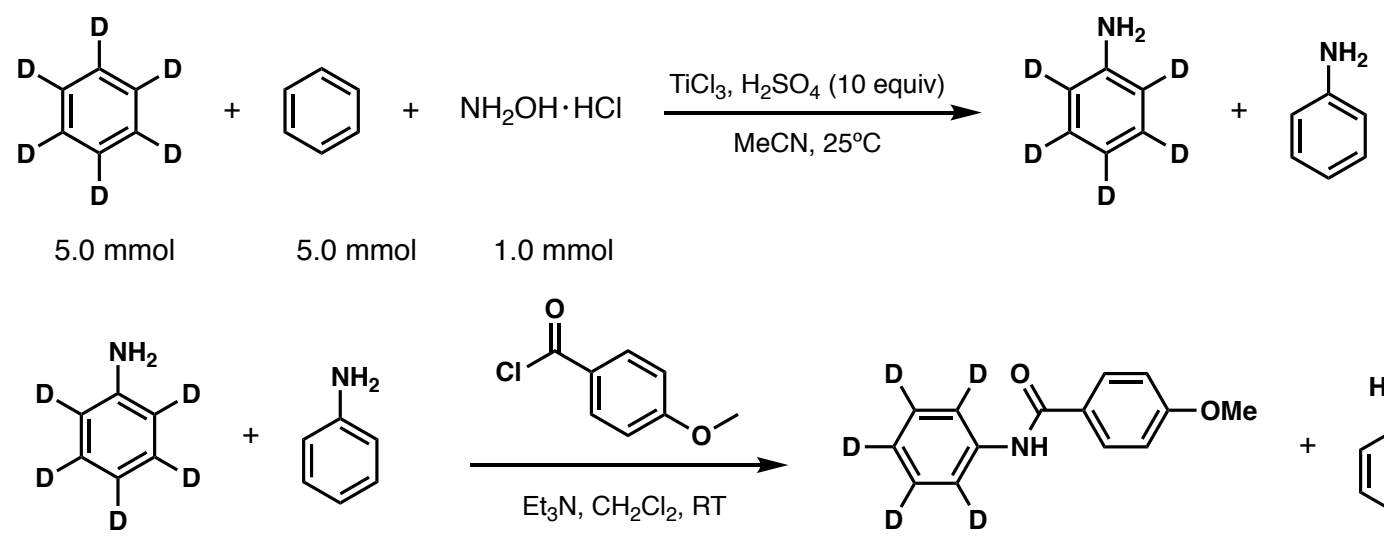<smiles>[2H]c1c([2H])c([2H])c(NC(=O)c2ccc(OC)cc2)c([2H])c1[2H]</smiles><smiles>COc1ccc(C(=O)Nc2ccccc2)cc1</smiles>

(crude)

Benzene $(5.0 \mathrm{mmol})$, benzene- $d_{6}(5.0 \mathrm{mmol})$, and hydroxylamine hydrochloride $(1.0 \mathrm{mmol})$ were suspended in acetonitrile $(2.0 \mathrm{~mL})$. The reaction was immersed in a rt $\left(25^{\circ} \mathrm{C}\right)$ water bath. Concentrated sulfuric acid $(10 \mathrm{mmol})$ was added dropwise, and the reaction was stirred open to air for $10 \mathrm{~min}$. After this time, the reaction was capped, and the headspace was purged with nitrogen for $1 \mathrm{~min}$. $\mathrm{TiCl}_{3}(12 \%$ in aqueous $\mathrm{HCl}$ ) was added via a syringe pump (addition rate: 4 $\mathrm{mL} / \mathrm{h}$ ) until the purple color of the titanium trichloride solution persisted for more than $10 \mathrm{~min}$. The reaction was stirred for an additional $10 \mathrm{~min}$ before the addition of saturated aqueous sodium citrate $(3 \mathrm{~mL})$. Sodium hydroxide ( $20 \%$ aqueous) was then added slowly until the solution turned basic. The reaction was subsequently extracted with ethyl acetate $(5 \times 10 \mathrm{~mL})$. The combined organic phases were dried over magnesium sulfate, and the solvent was removed via rotary evaporation.

The resulting crude mixture of anilines was taken up in dry $\mathrm{CH}_{2} \mathrm{Cl}_{2}(5 \mathrm{~mL})$. Triethylamine (1.5 $\mathrm{mmol})$ was added, followed by $p$-anisolyl chloride $(1.0 \mathrm{mmol})$. The reaction was stirred overnight at rt. The reaction was then quenched by the addition of saturated sodium bicarbonate and stirred for $30 \mathrm{~min}$ at rt. The reaction was washed with water $(5 \mathrm{~mL})$, and the aqueous layer was backextracted with $\mathrm{CH}_{2} \mathrm{Cl}_{2}(2 \times 5 \mathrm{~mL})$. The combined organic phases were then dried over magnesium sulfate, concentrated via rotary evaporation, and purified by preparative TLC.

Based on the integration of the proton signals in the ${ }^{1} \mathrm{H}$ NMR spectrum, the kinetic isotope effect was determined to be $\sim 1.0$ (Figure S1). The experiment was repeated 2 additional times with identical results. 


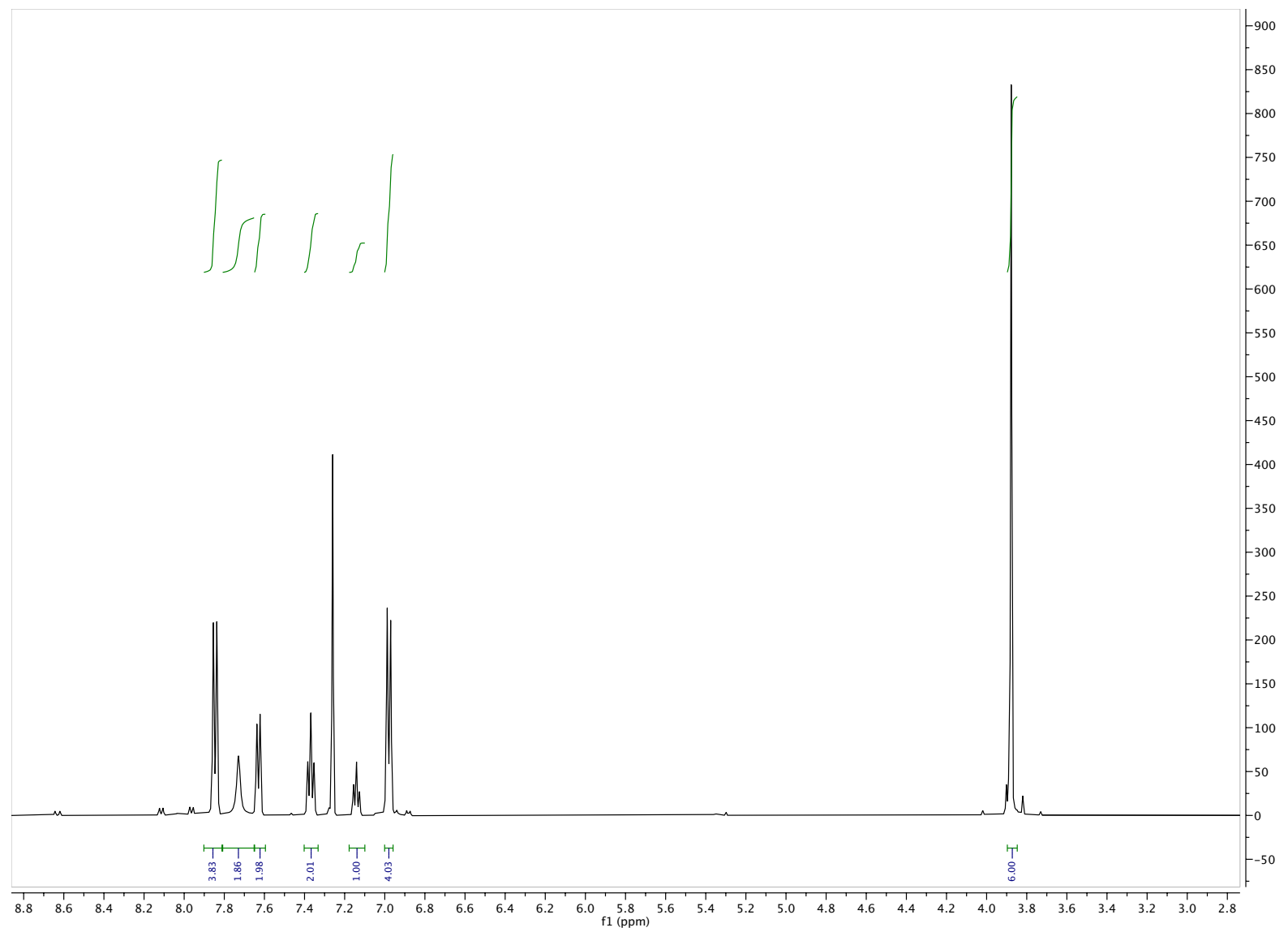

Figure S1. $1 \mathrm{H}$ NMR of $d_{5}$-4-methoxy- $N$-phenylbenzamide and 4-methoxy- $N$-phenylbenzamide from KIE experiment. 


\section{References}

1. Rössler, S. L.; Jelier, B. J.; Tripet, P. F.; Shemet, A.; Jeschke, G.; Togni, A.; Carreira, E. M. Angew. Chem. Int. Ed. 2019, 58, 526-531.

2. Legnani, L.; Prina Cerai, G.; Morandi, B. ACS Catal. 2016, 6, 8162-8165.

3. Liu, J.; Wu, K.; Shen, T.; Liang, Y.; Zou, M.; Zhu, Y.; Li, X.; Li, X.; Jiao, N. Chem. Eur. J. 2017, 23, 563-567.

4. Friis, S. D.; Andersen, T. L.; Skrydstrup, T. Org. Lett. 2013, 15, 1378-1381. 


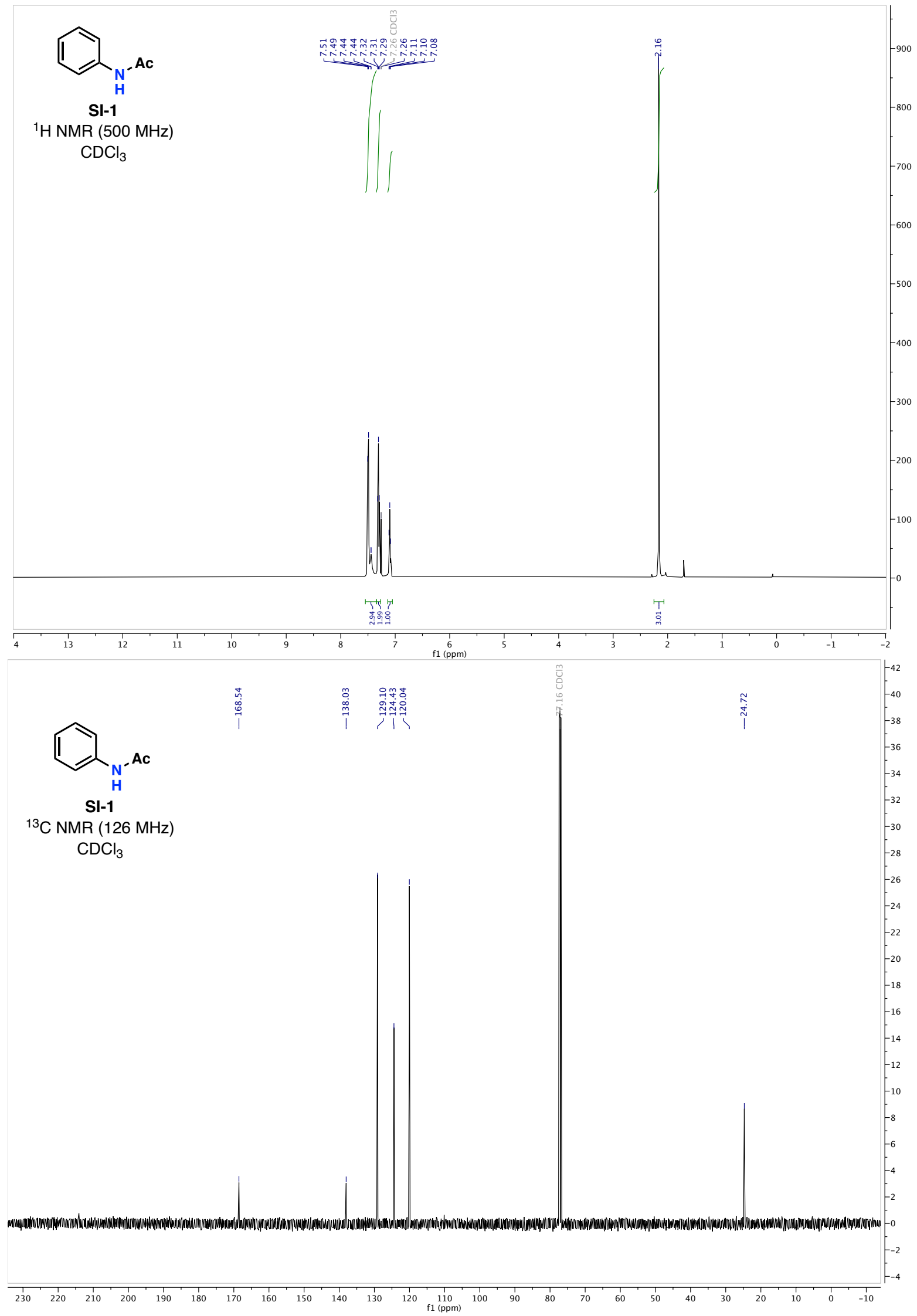

SI-33 


<smiles>COc1ccccc1N</smiles>



2b

${ }^{13} \mathrm{C}$ NMR $(126 \mathrm{MHz})$

$\mathrm{CDCl}_{3}$

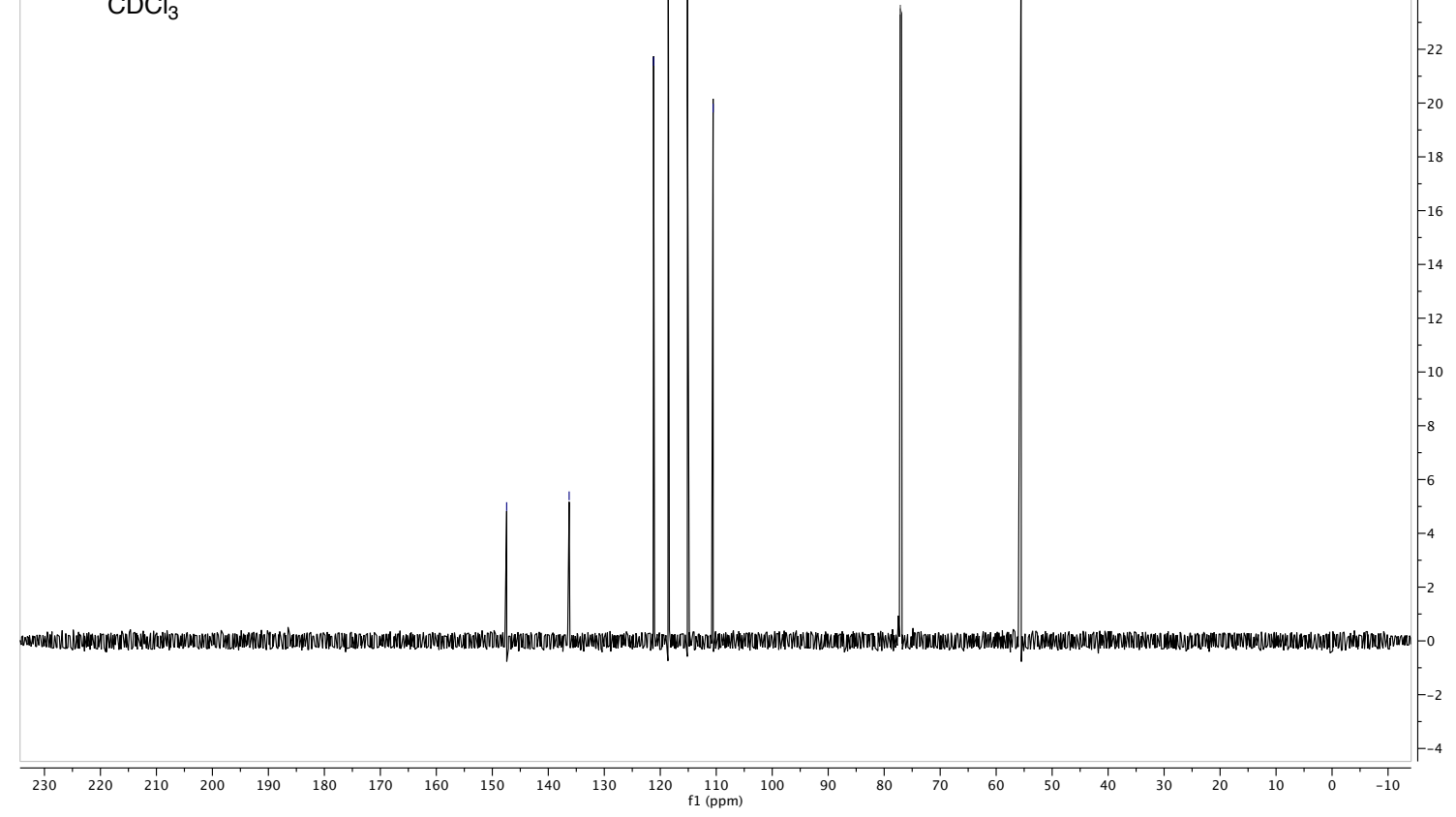






SI-35 




SI-36 


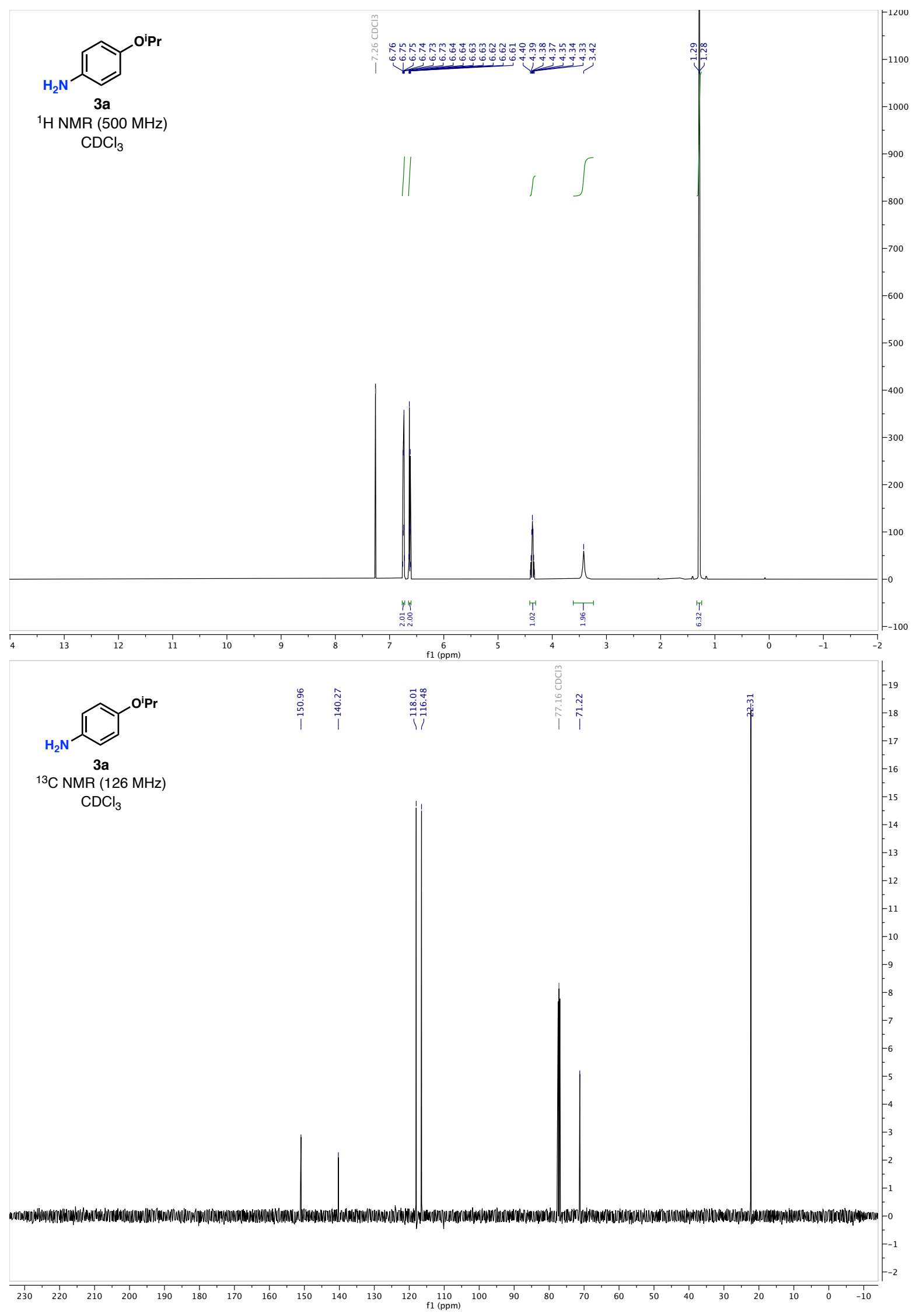

SI-37 




SI-38 


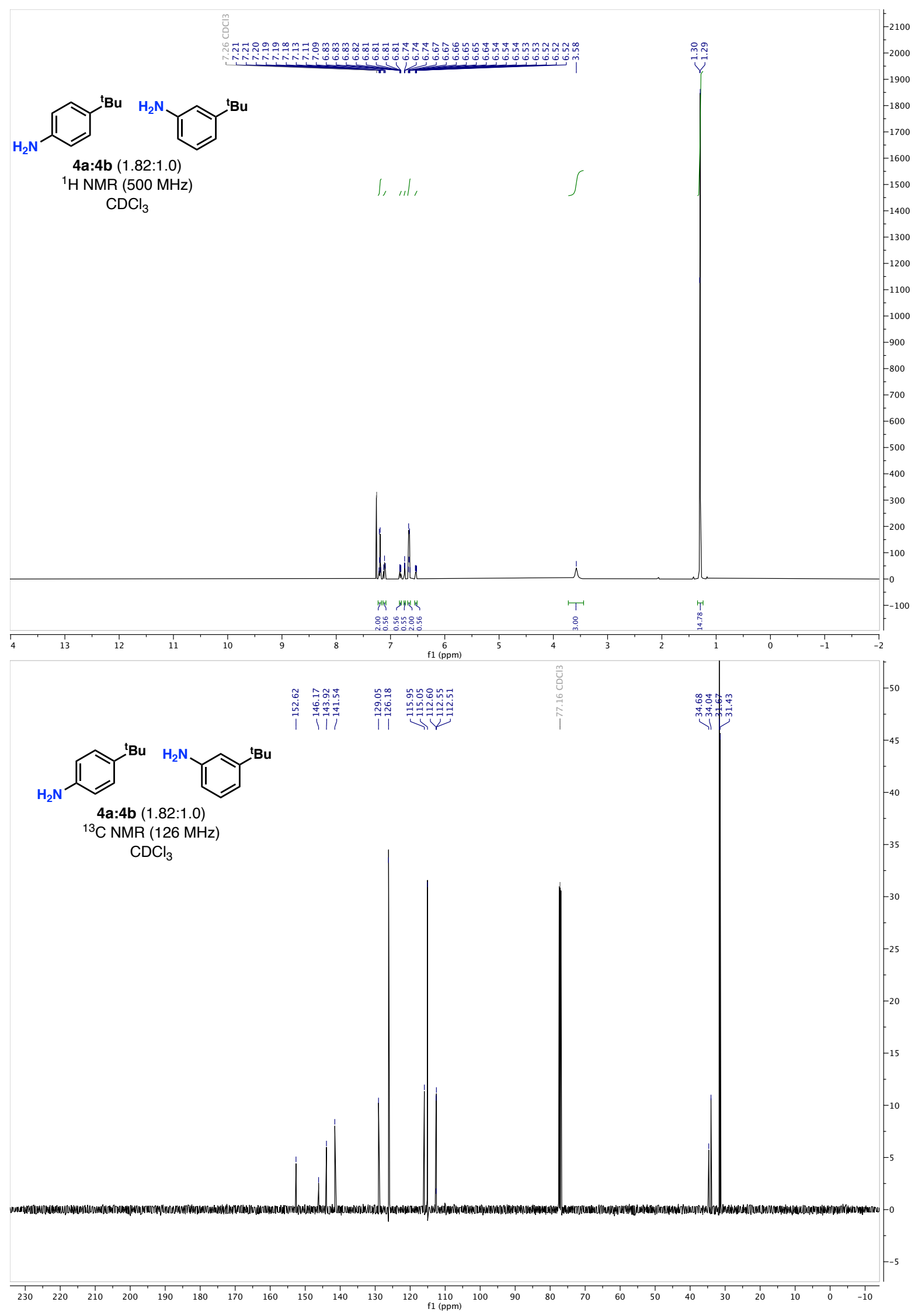

SI-39 


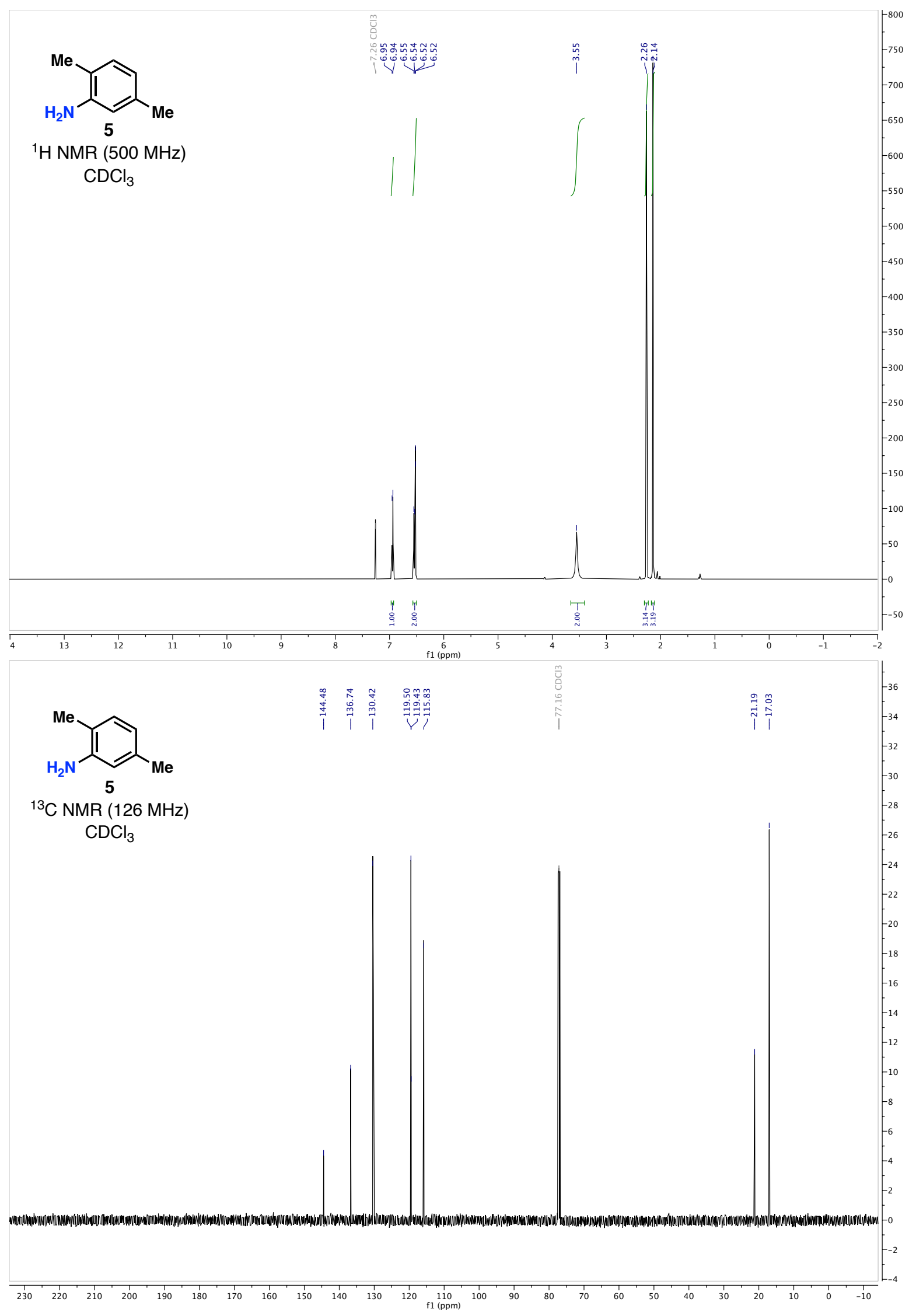

SI-40 


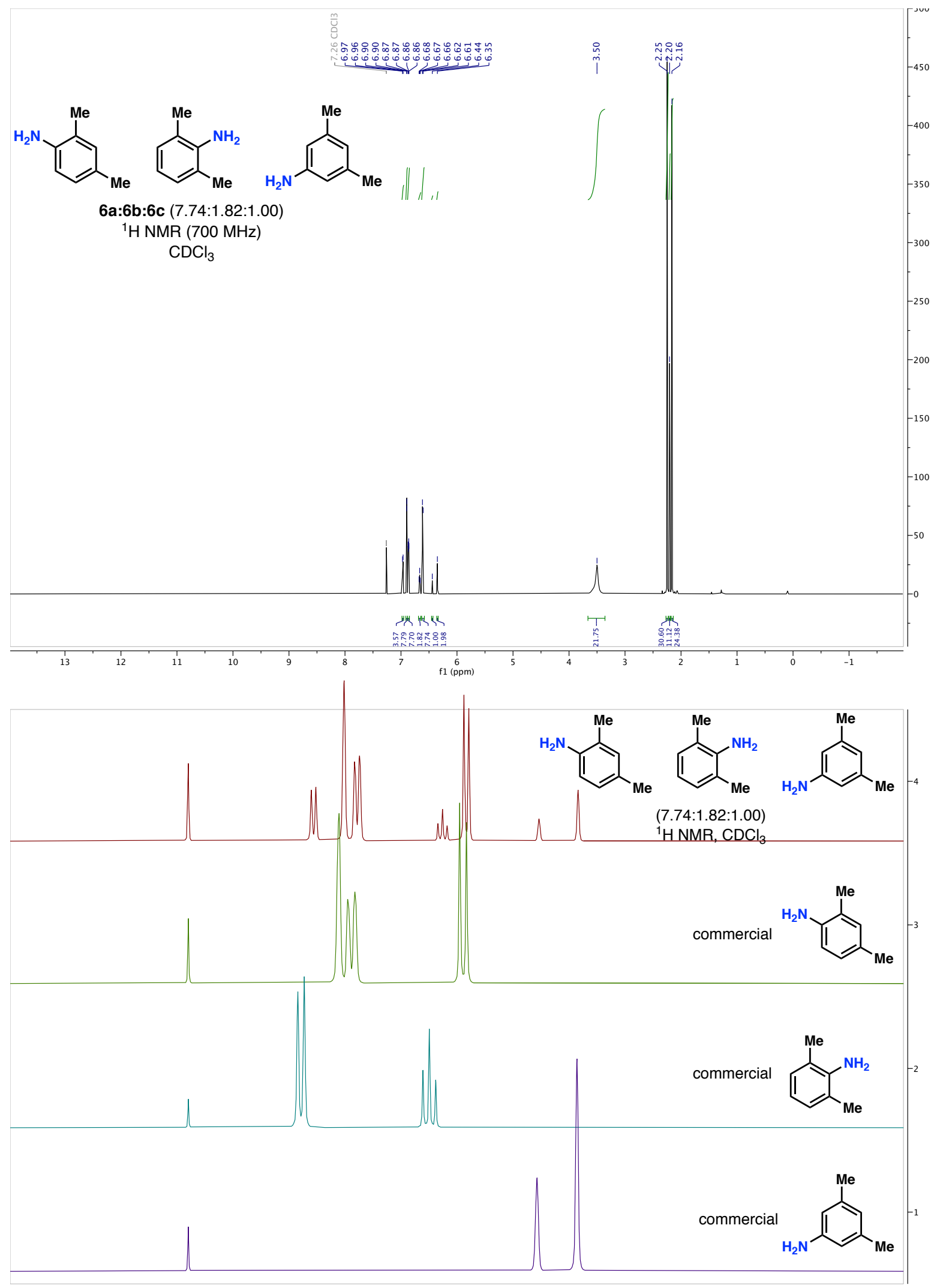





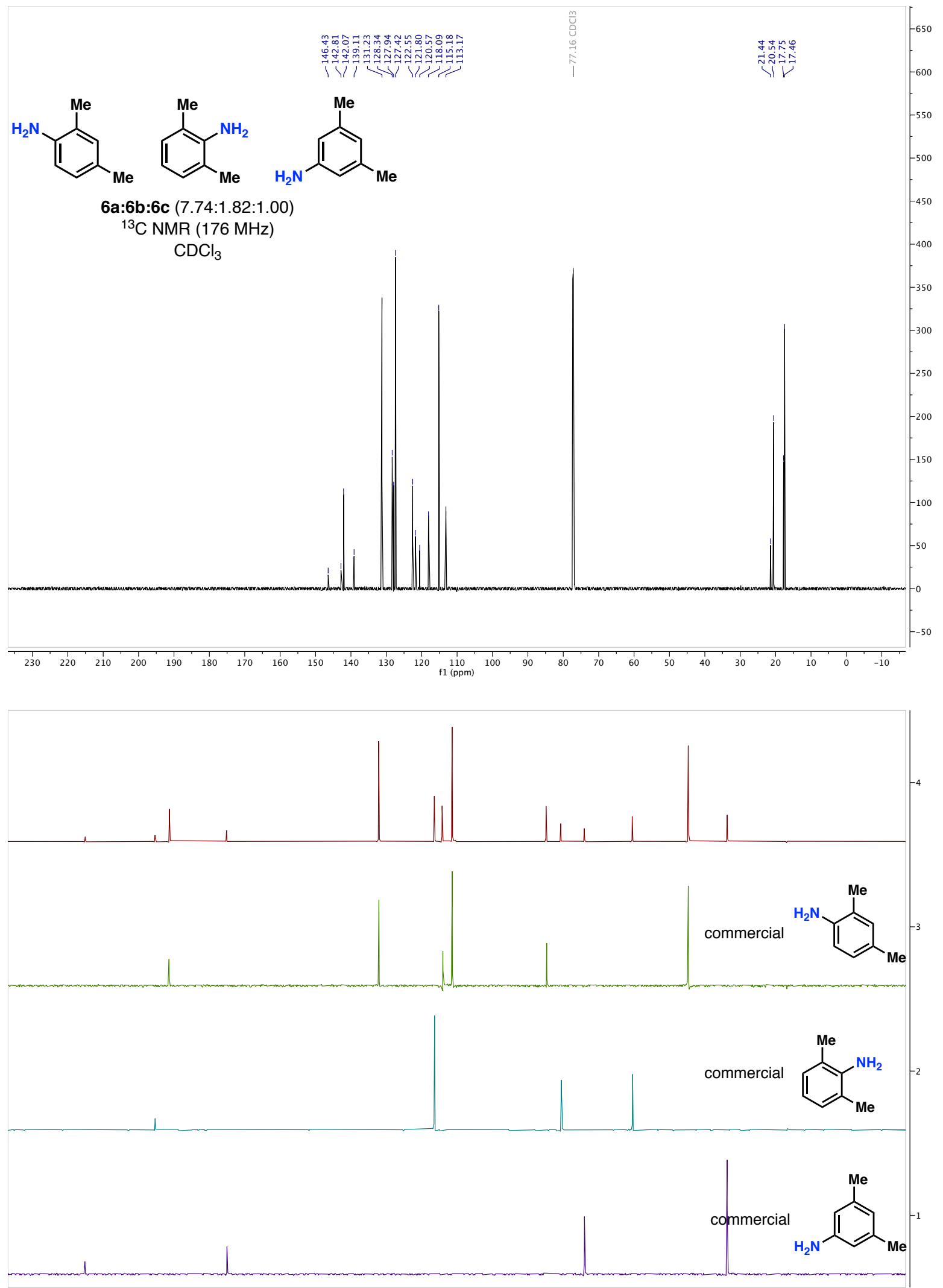

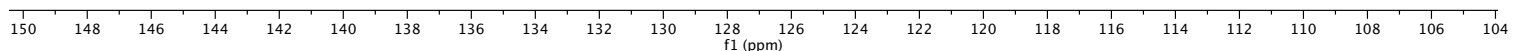














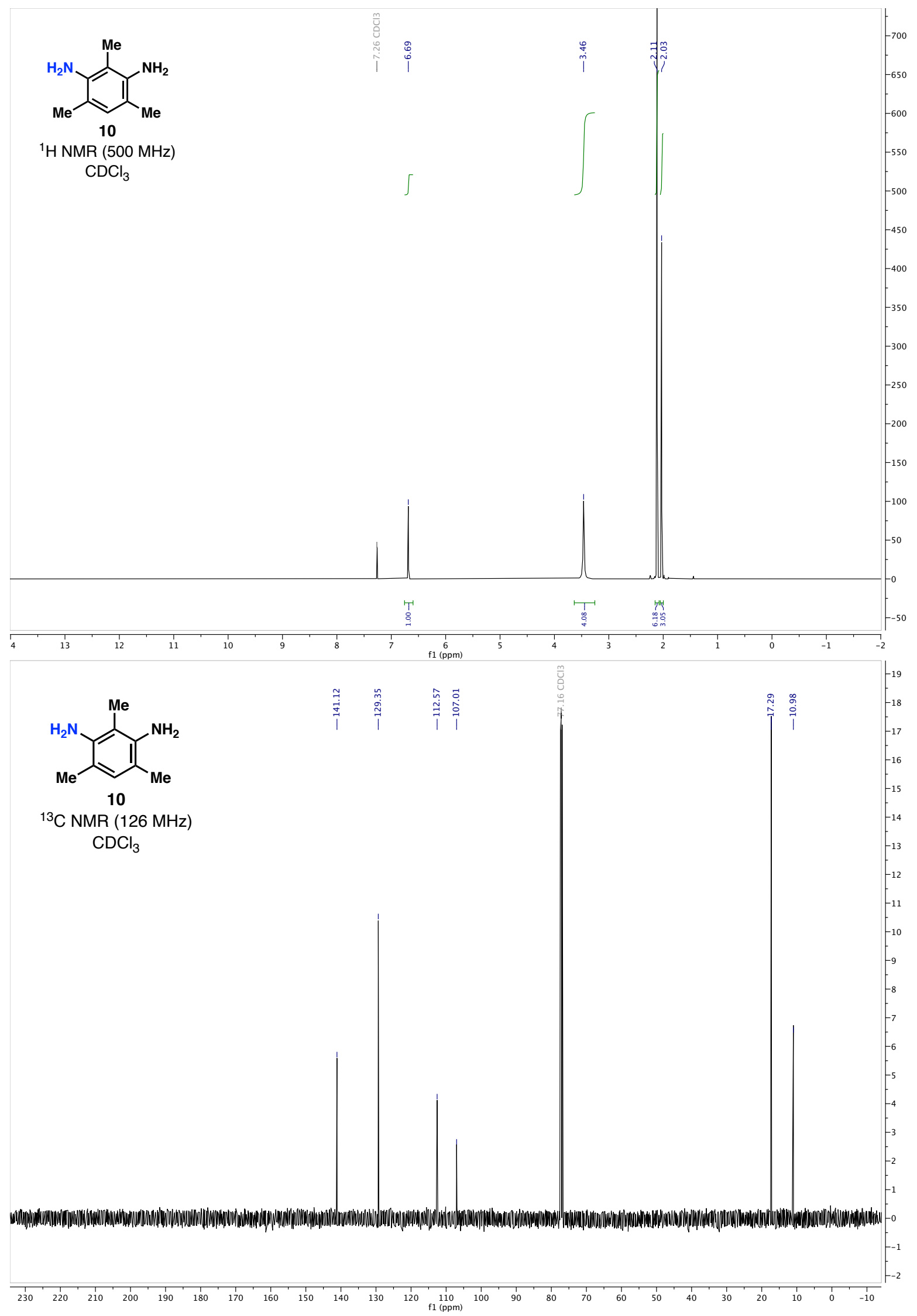

SI-45 









SI-47 


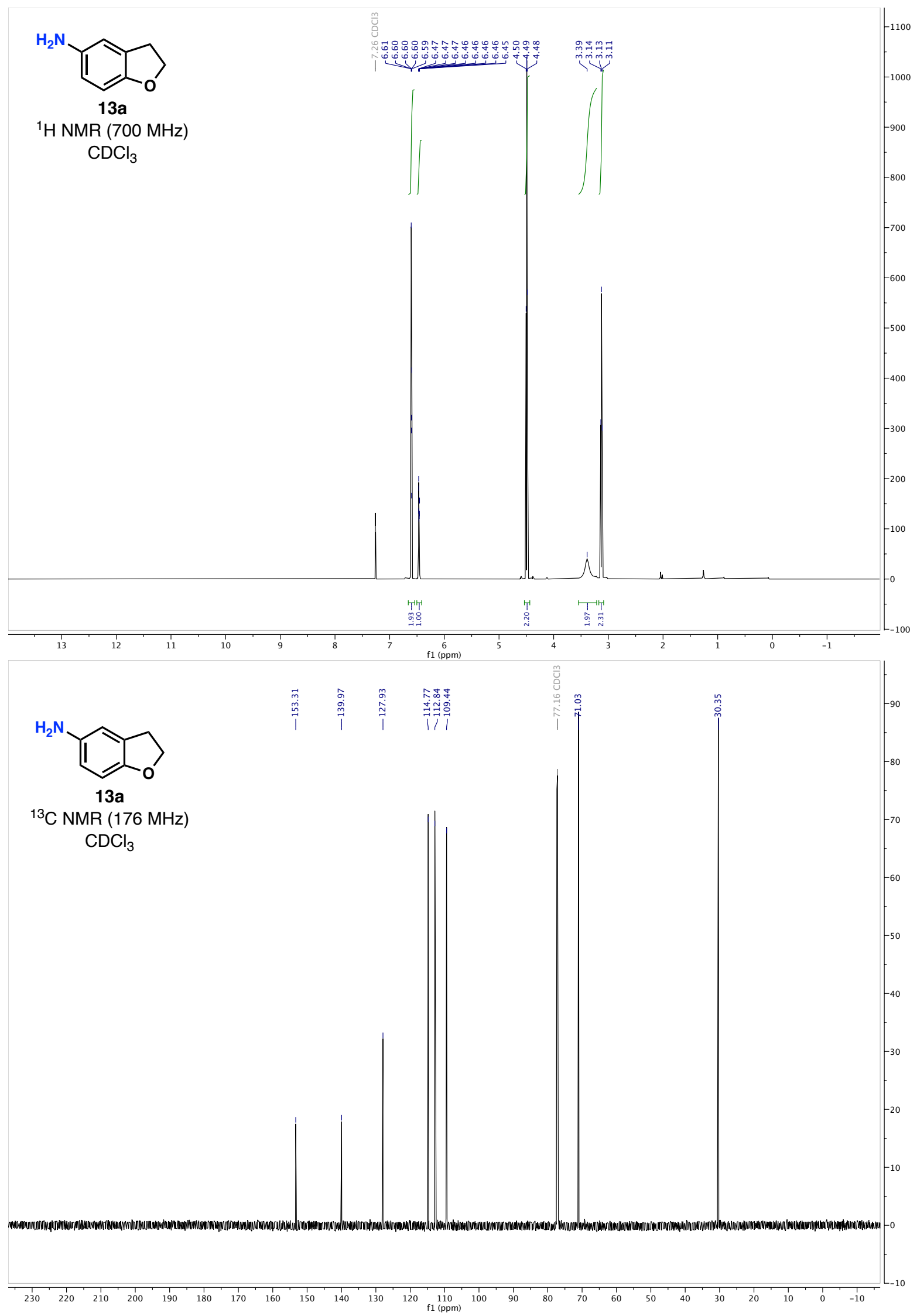









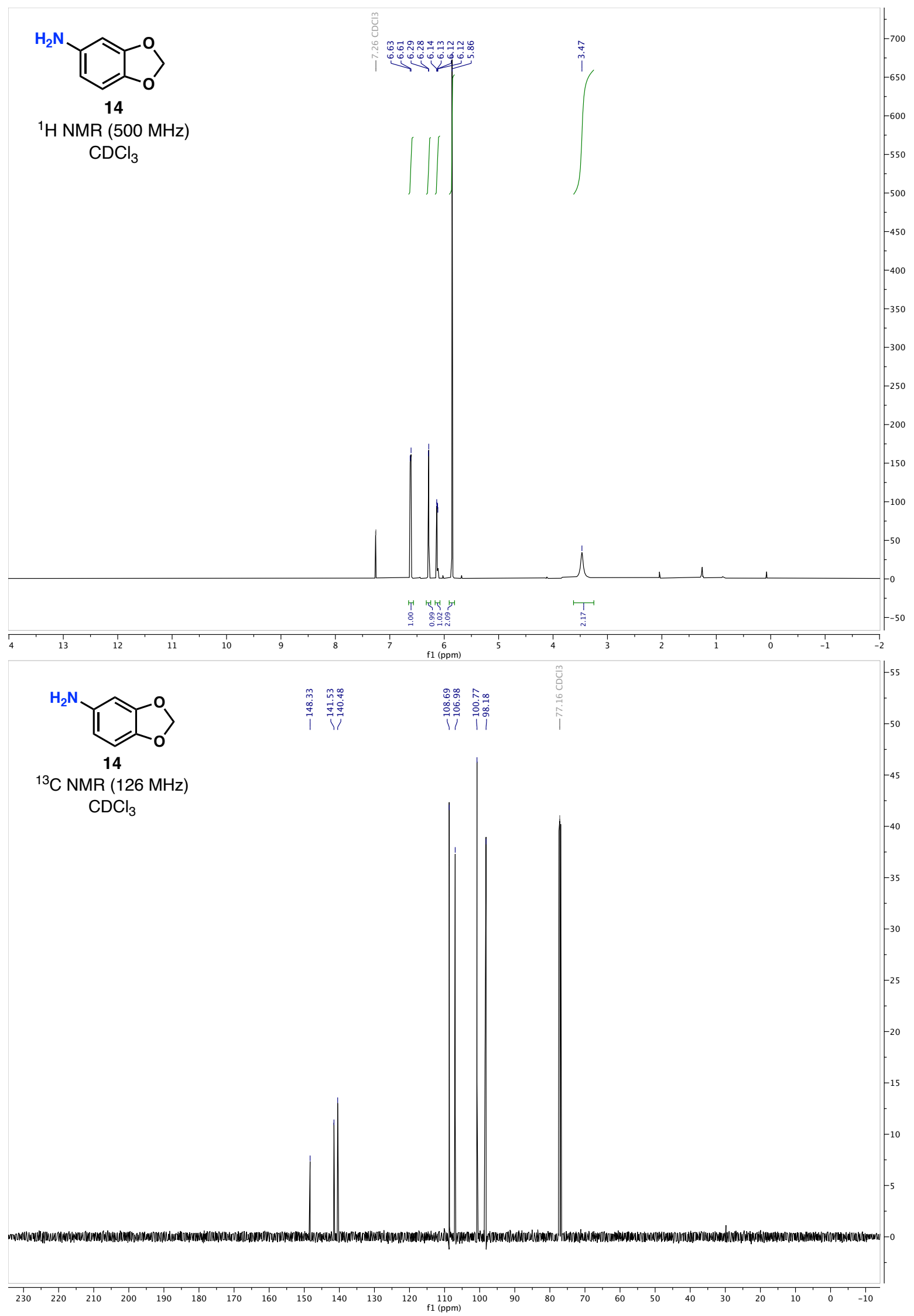

SI-50 


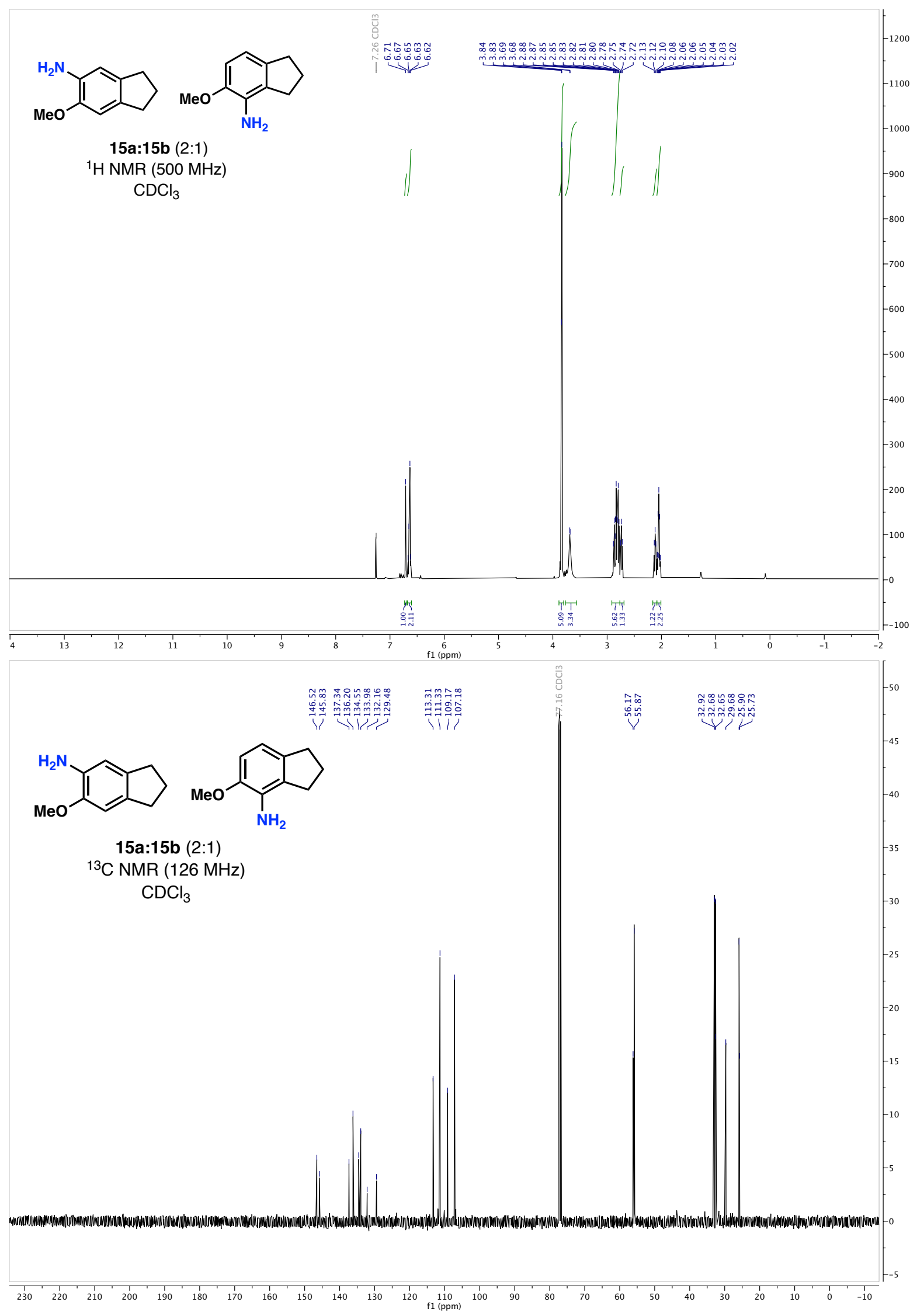




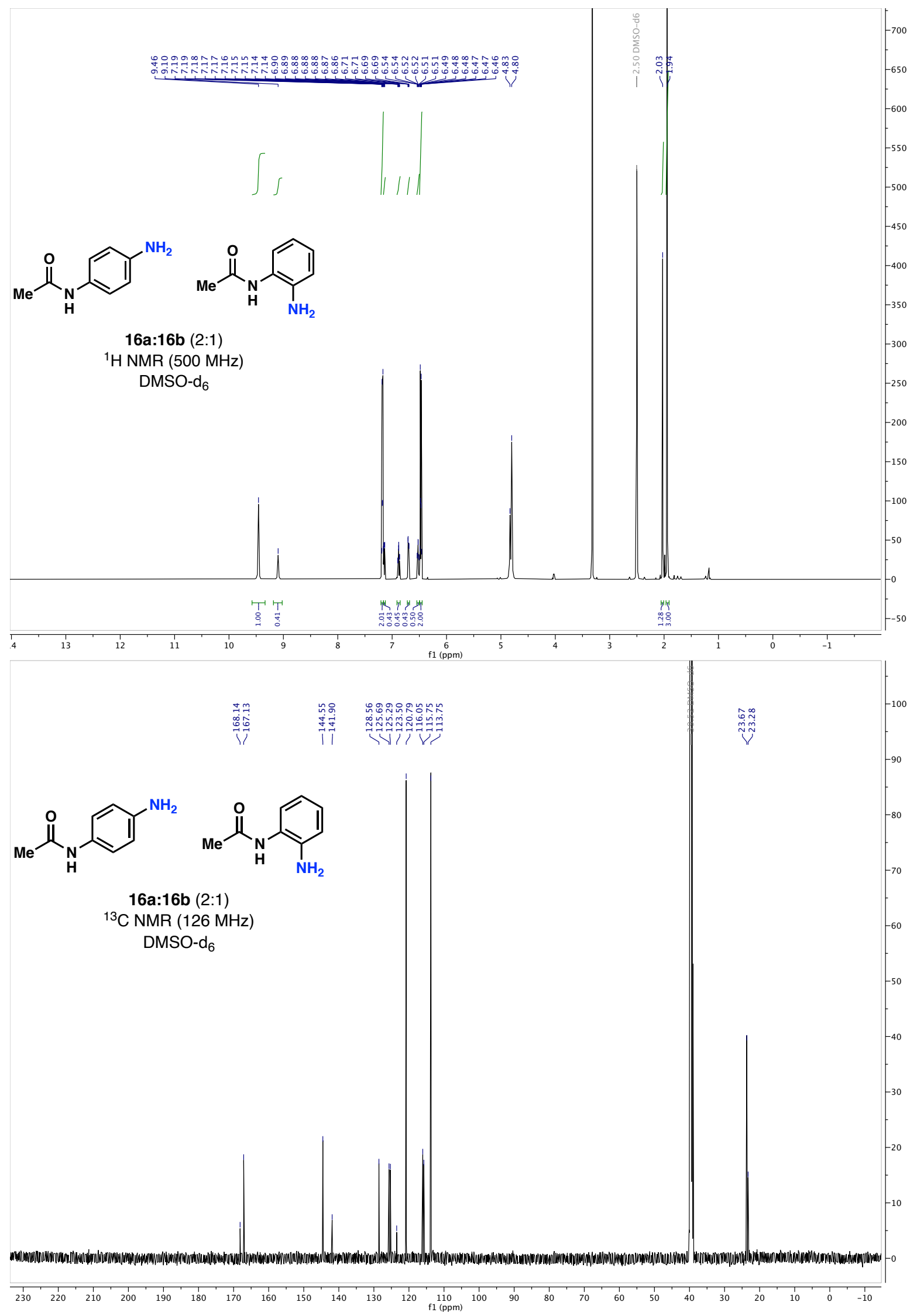






SI-53 




SI-54 




SI-55 




SI-56 




SI-57 


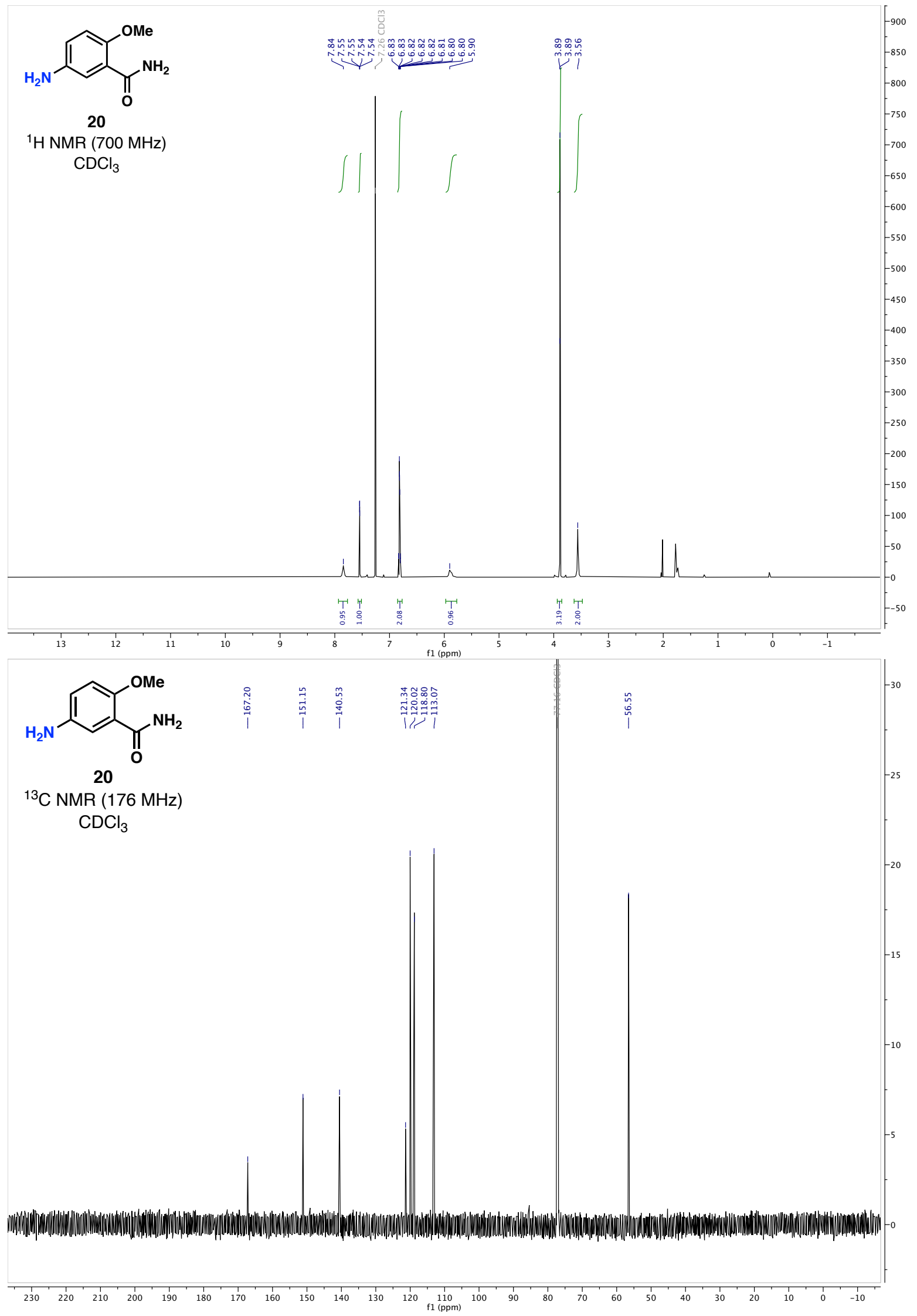

SI-58 


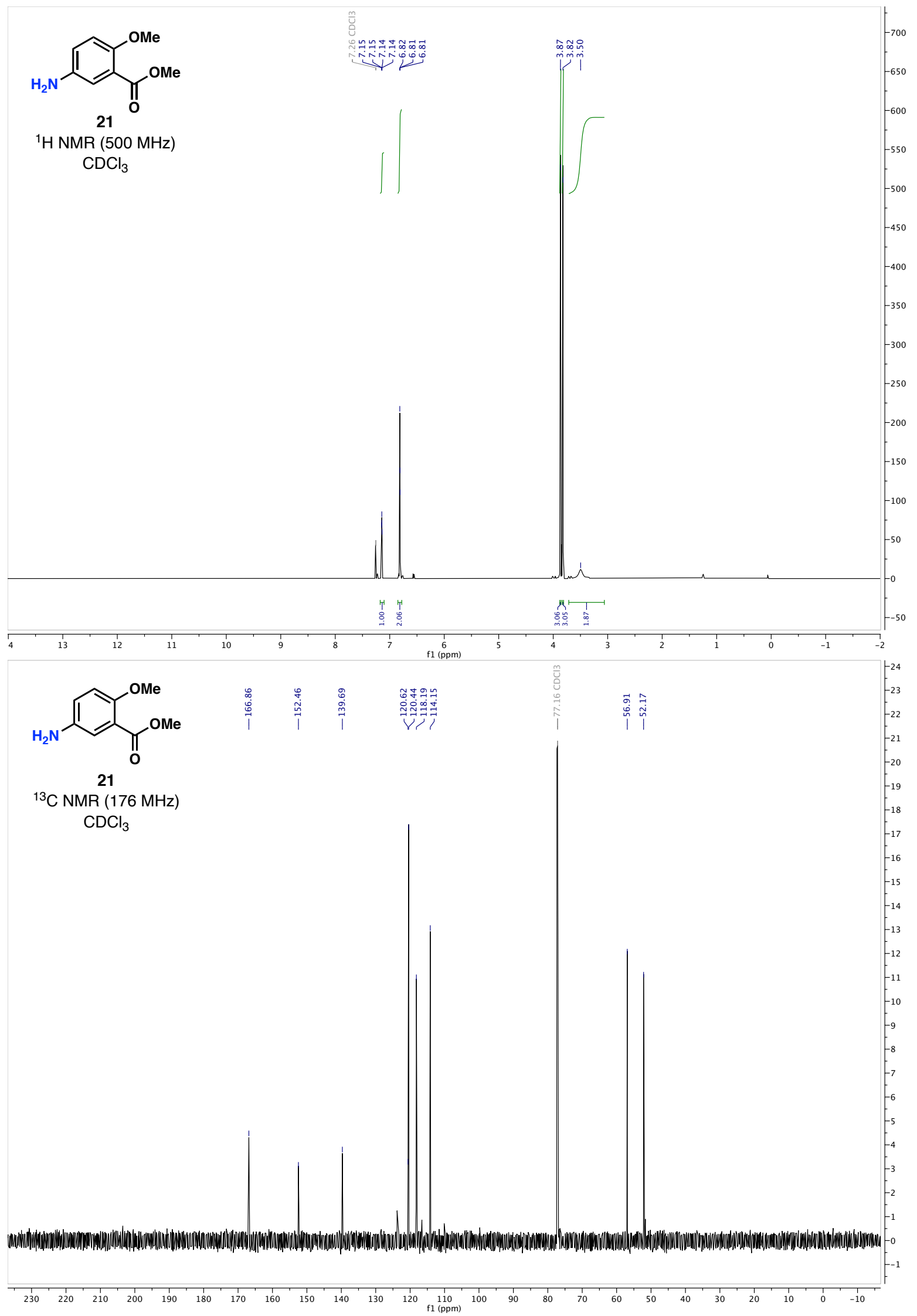

SI-59 


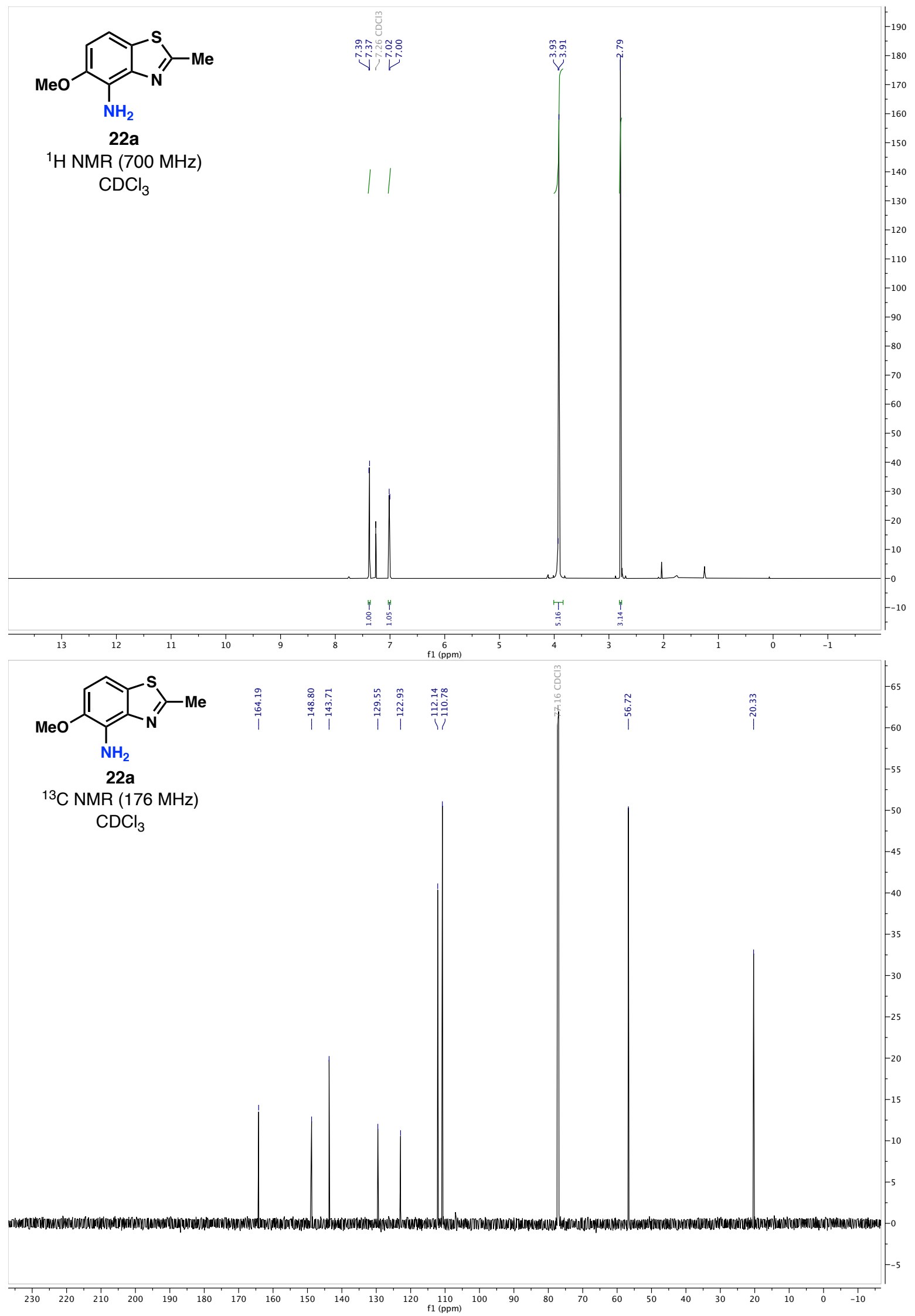




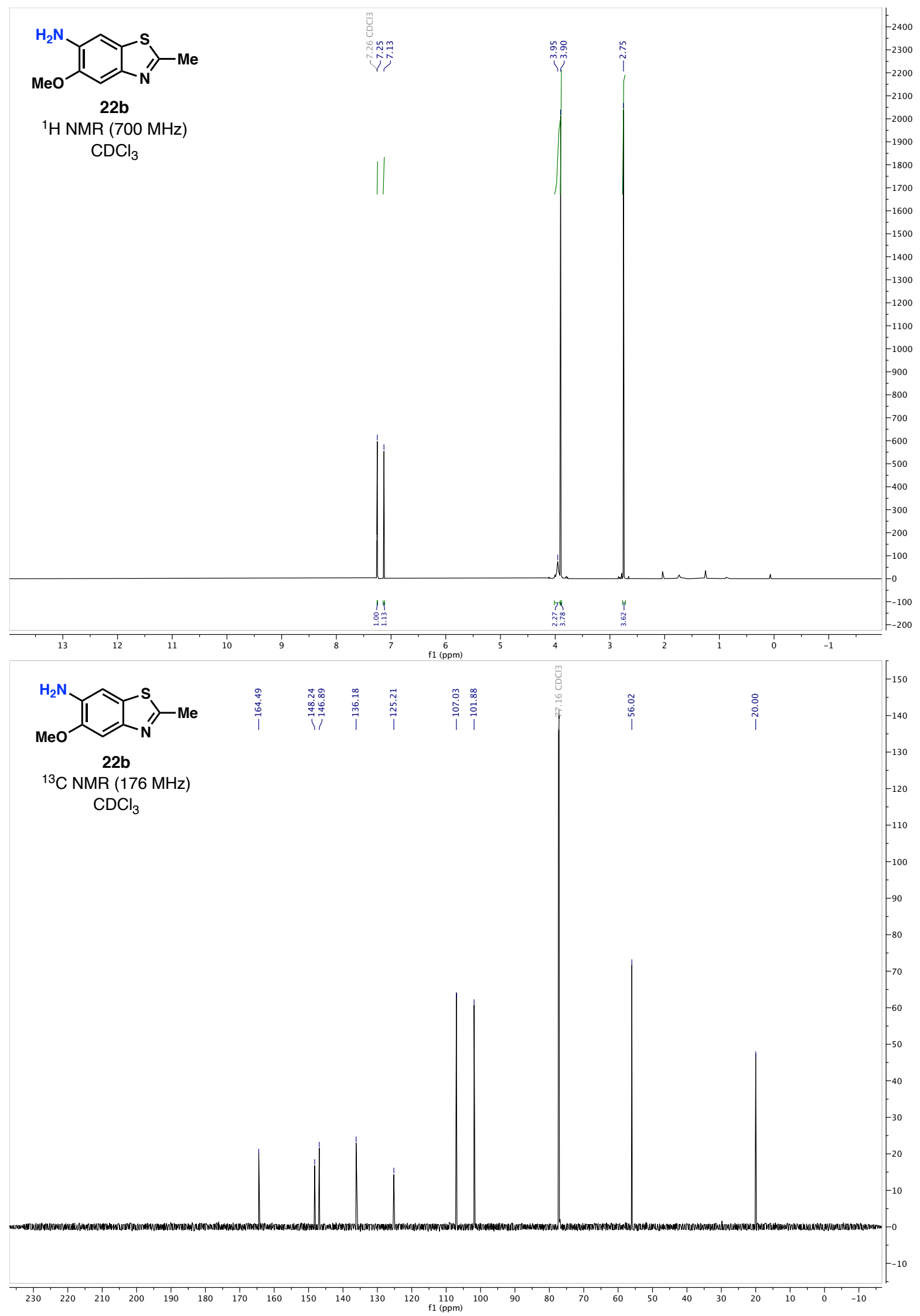

SI-61 


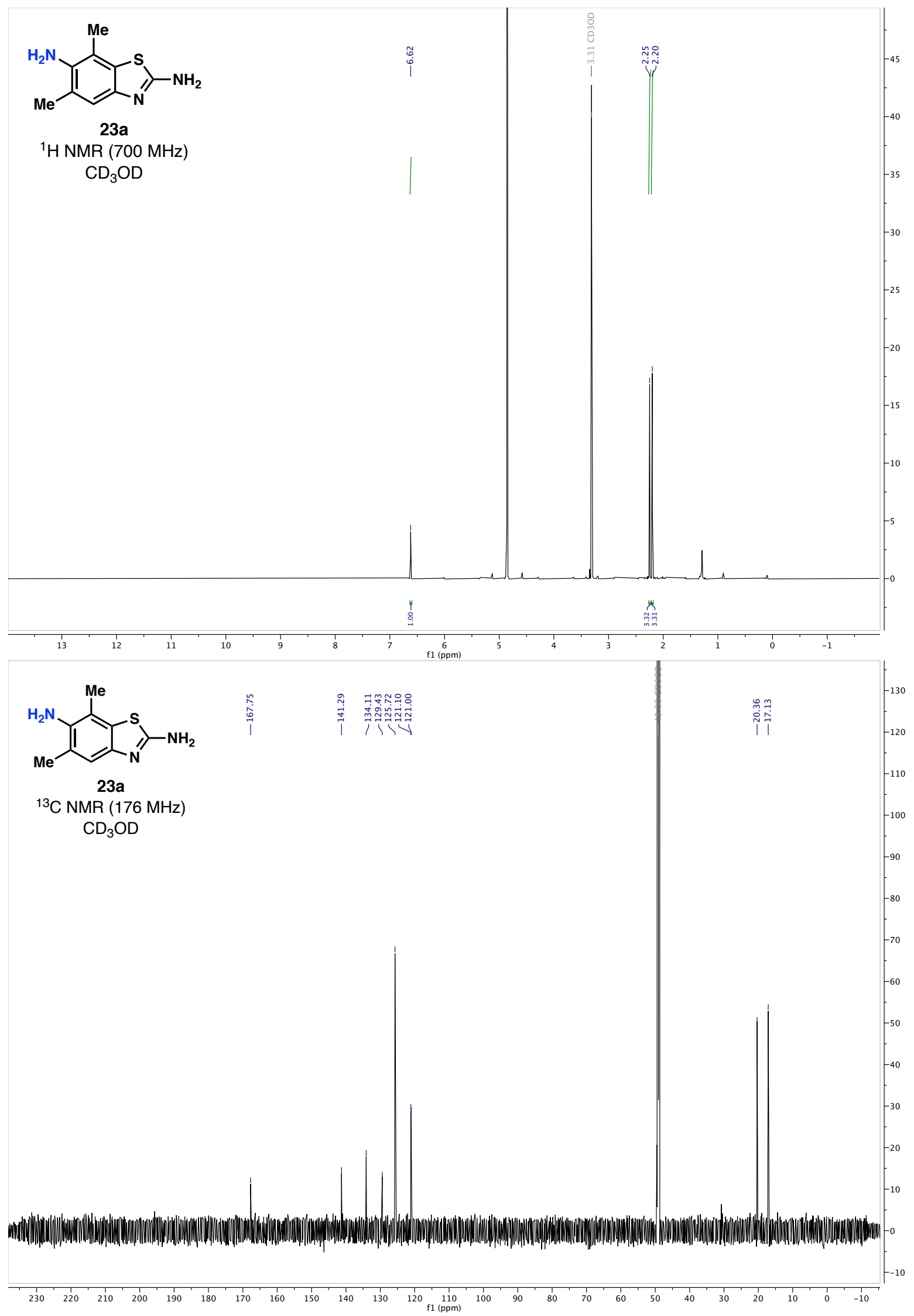

SI-62 


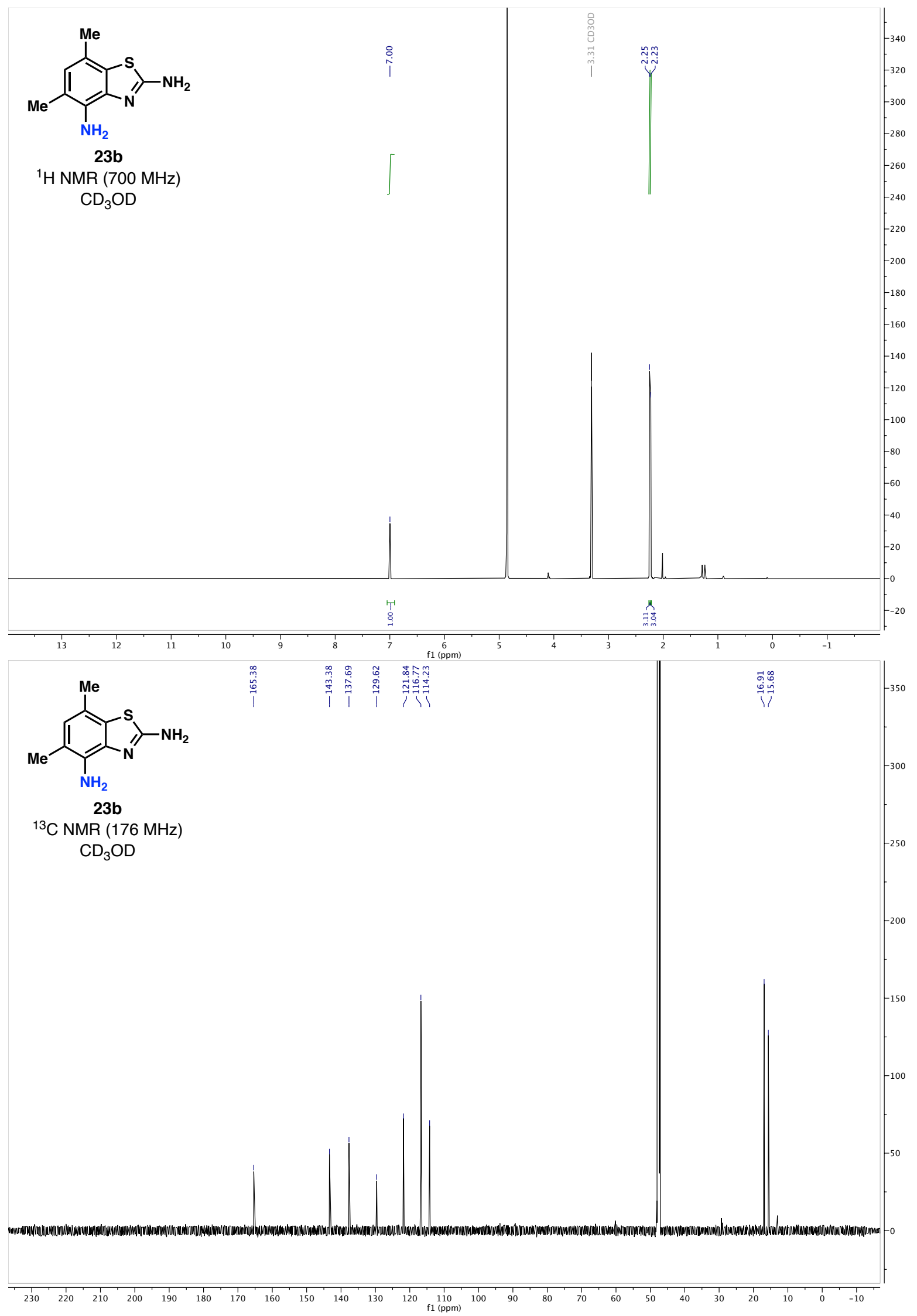








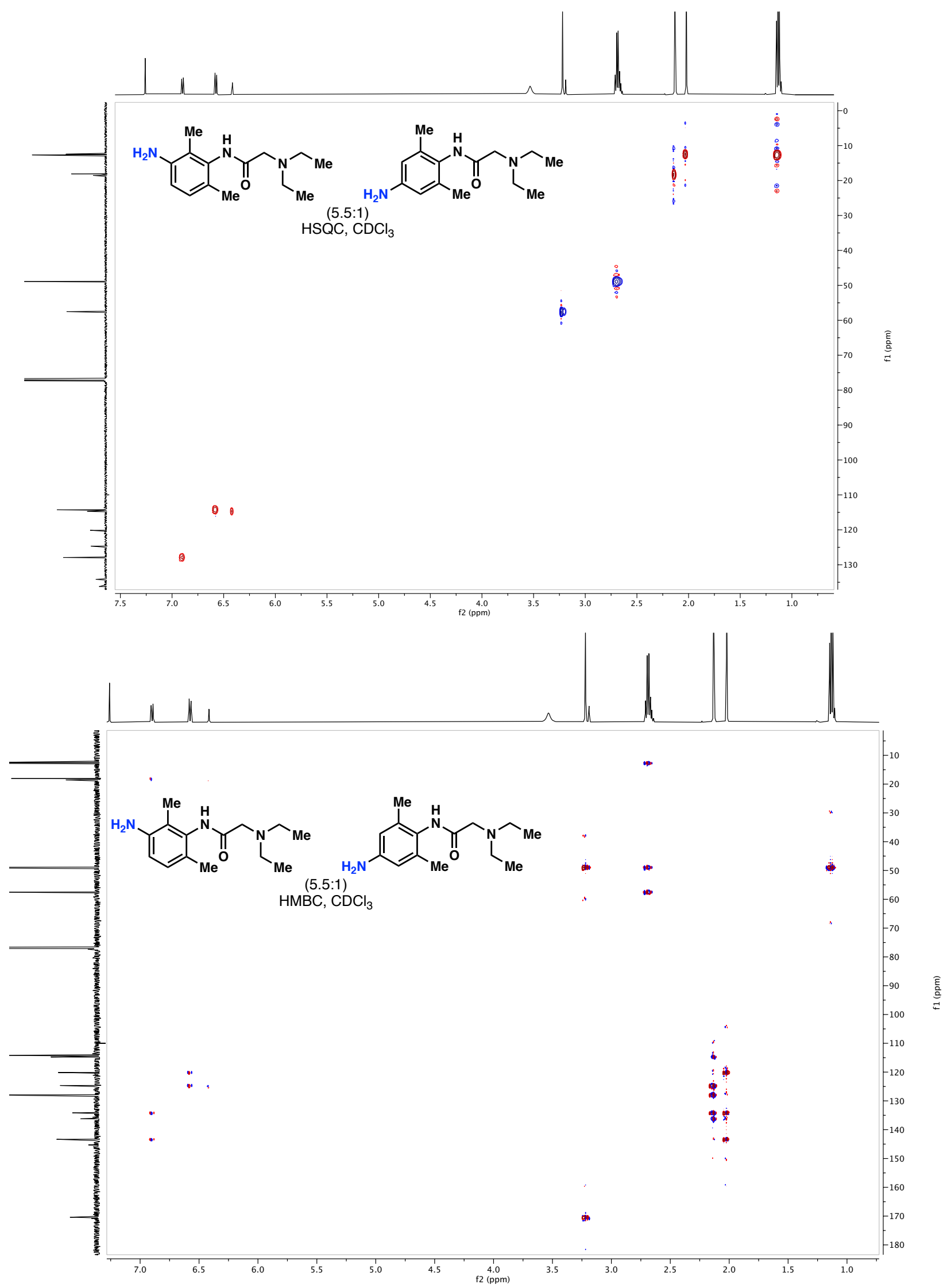


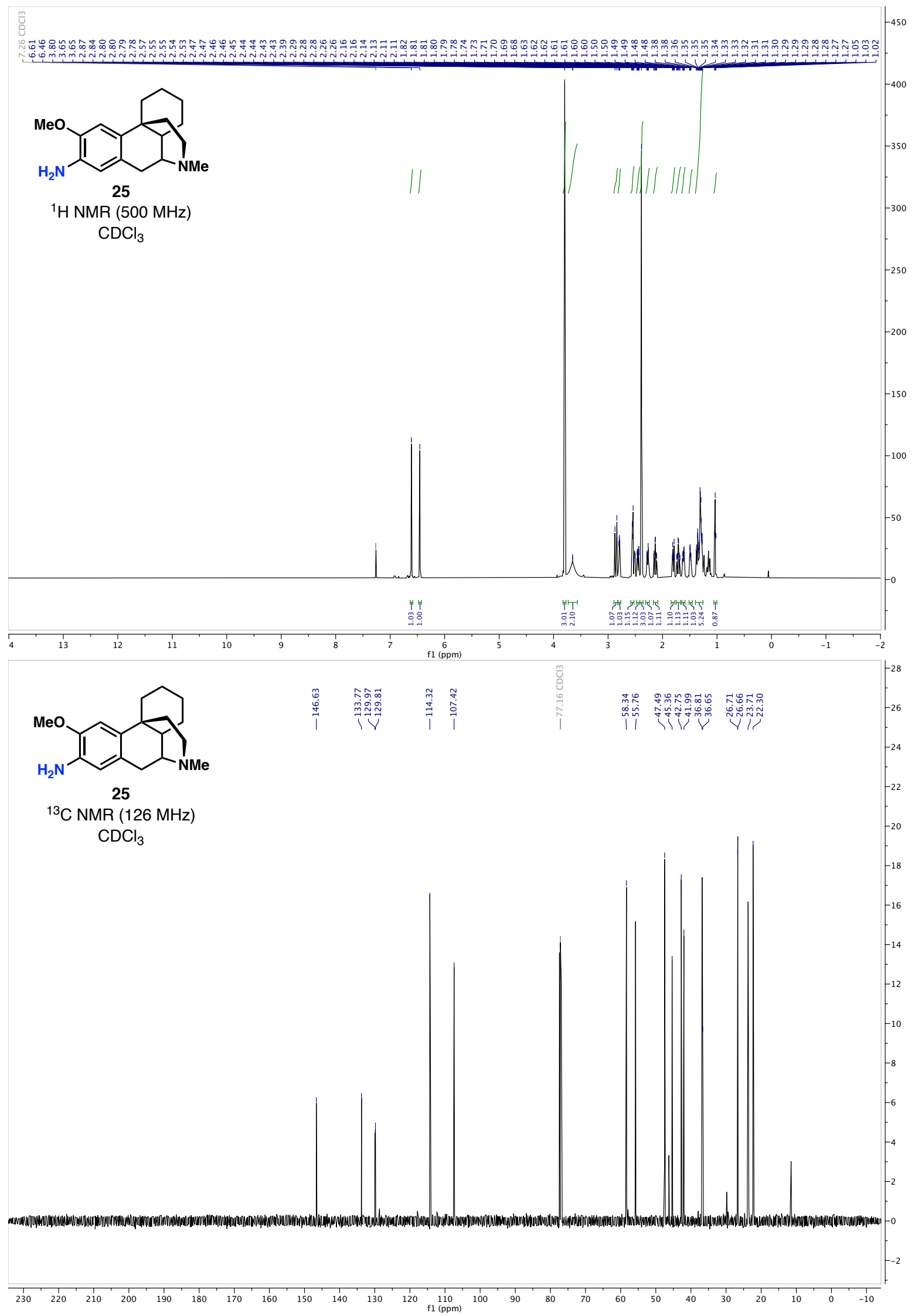




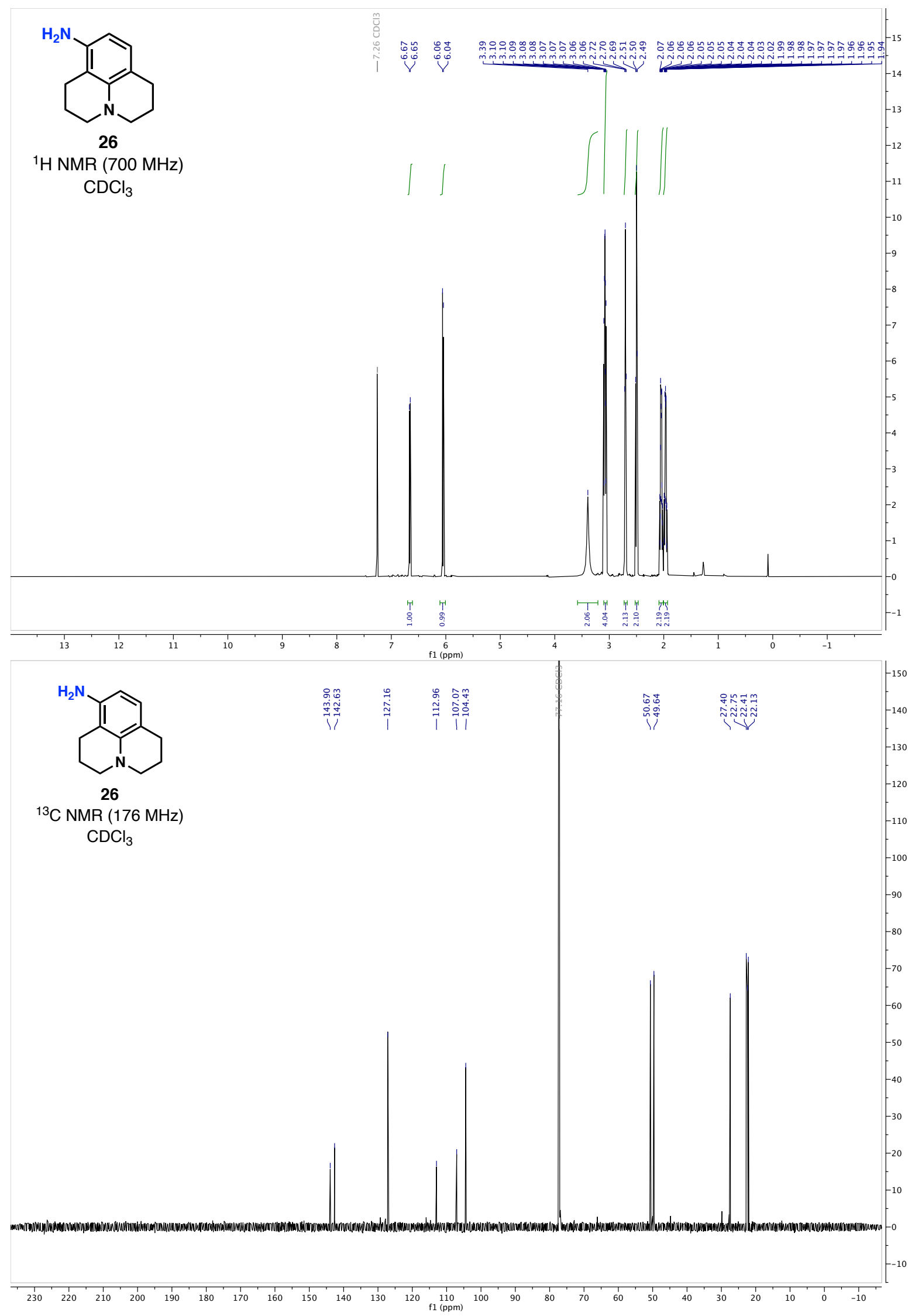



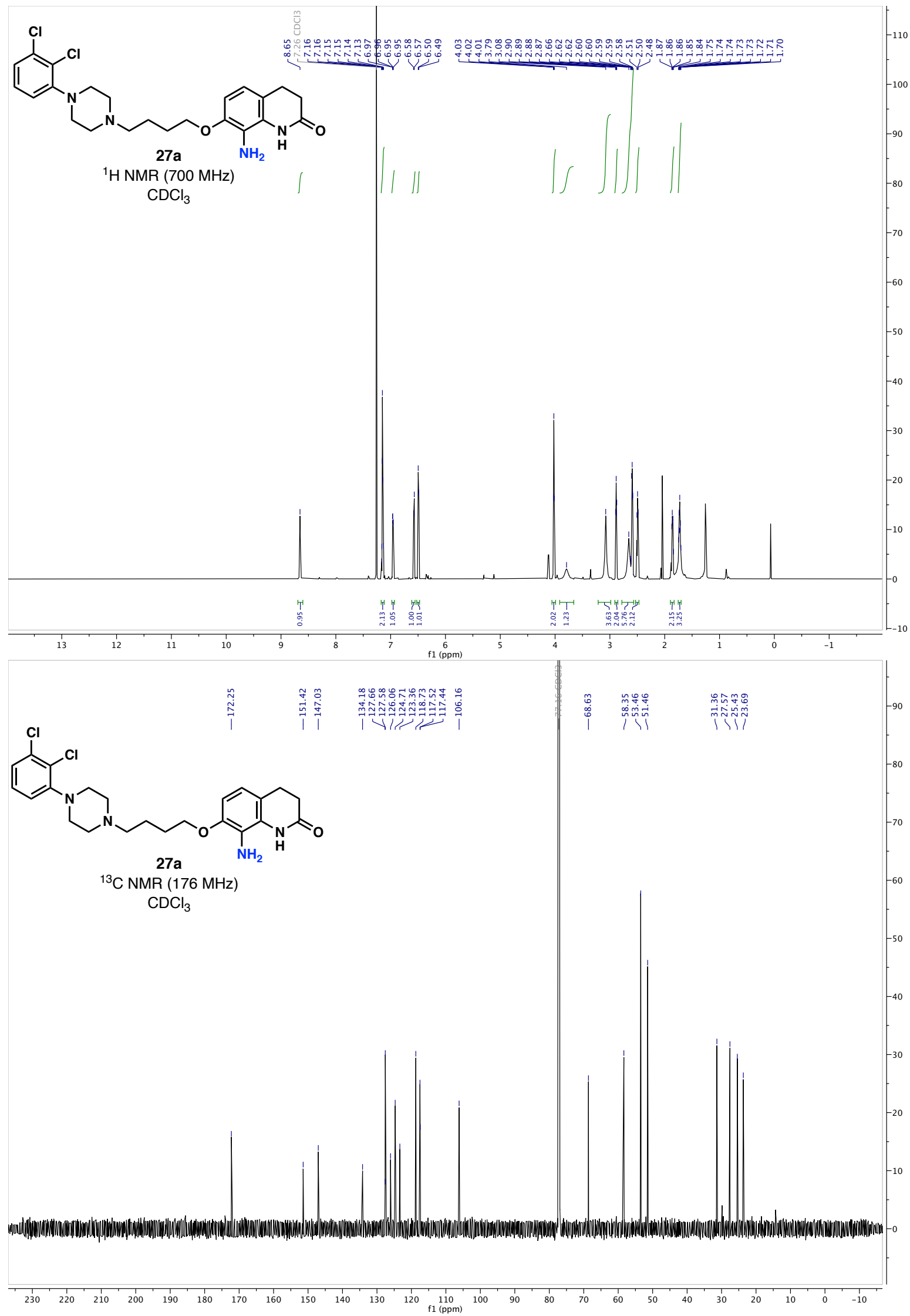


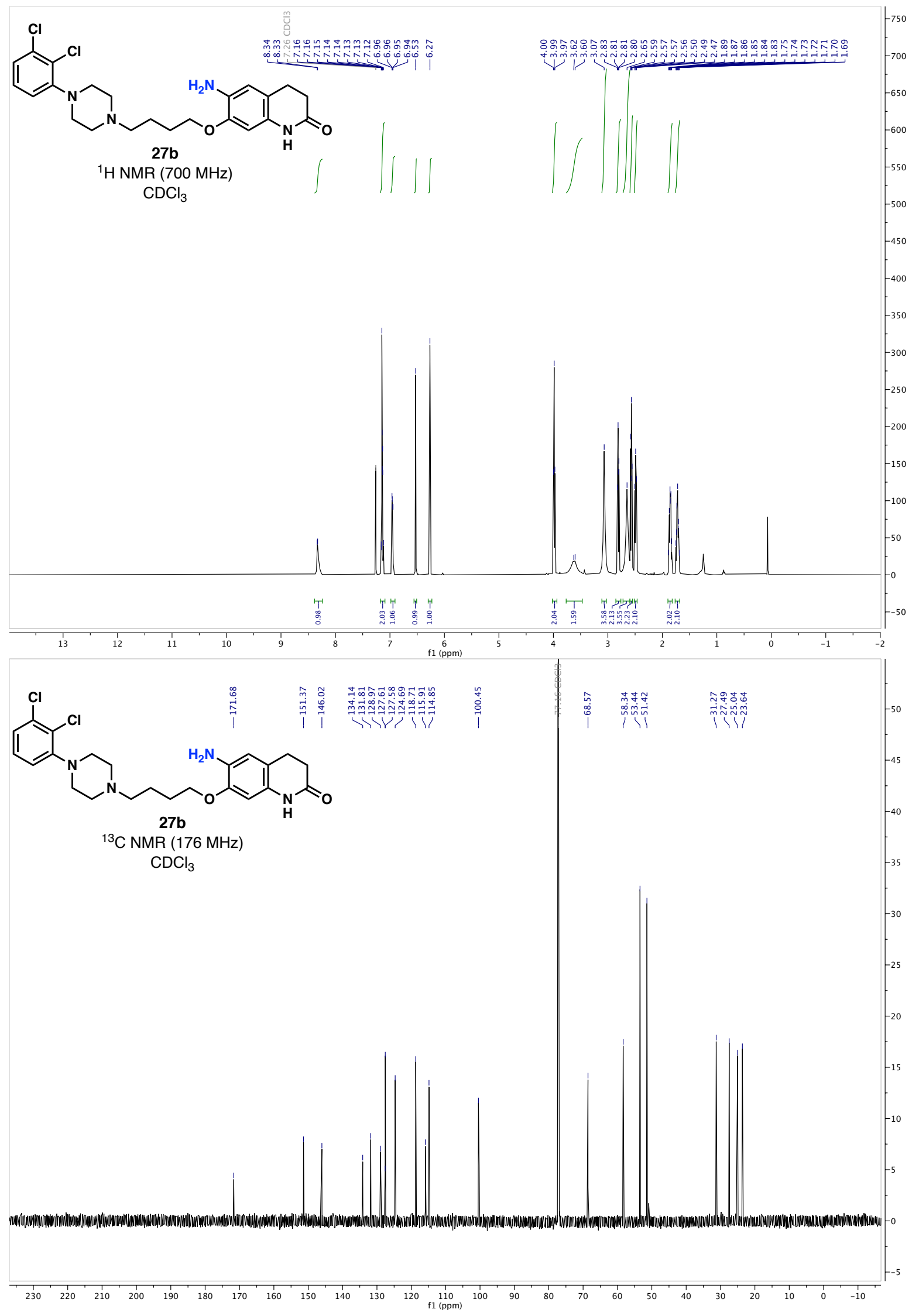

